\title{
The Implication of Spatial Statistics in Human Mesenchymal Stem Cell Response to Nanotubular Architectures
}

This article was published in the following Dove Press journal: International Journal of Nanomedicine

\begin{abstract}
Alexander J Steeves $\mathbb{D}^{1,2}$
William Ho (D) ${ }^{1,2, *}$

Maria Chiara Munisso (D) ${ }^{3, *}$

David J Lomboni (iD) ${ }^{1,2}$

Enara Larrañaga (iD ${ }^{4}$

Sidney Omelon ${ }^{1,5}$

Elena Martínez (D) 4,7

Davide Spinello (D)

Fabio Variola (iD) ${ }^{1,2,8,9}$

'Faculty of Engineering, Department of Mechanical Engineering, University of Ottawa, Ottawa, ON, Canada; ${ }^{2}$ OttawaCarleton Institute for Biomedical Engineering, Ottawa, Canada; ${ }^{3}$ Department of Plastic and Reconstructive Surgery, Kansai Medical University, Moriguchi, Japan; ${ }^{4}$ Institute for Bioengineering of Catalonia (IBEC), The Barcelona Institute of Science and Technology (BIST), Barcelona, Spain; ${ }^{5}$ Faculty of Engineering, Department of Mining and Materials Engineering, McGill University, Montreal, QC, Canada; ${ }^{6}$ Centro de Investigación Biomédica en Red (CIBER), Madrid, Spain; ${ }^{7}$ Department of Electronics and Biomedical Engineering, University of Barcelona, Barcelona, Spain; ${ }^{8}$ Faculty of Medicine, Department of Cellular and Molecular Medicine, University of Ottawa, Ottawa, ON, Canada; ${ }^{9}$ Children's Hospital of Eastern Ontario (CHEO), Ottawa, ON, Canada
\end{abstract}

*These authors contributed equally to this work

Correspondence: Fabio Variola Email fabio.variola@uottawa.ca
Introduction: In recent years there has been ample interest in nanoscale modifications of synthetic biomaterials to understand fundamental aspects of cell-surface interactions towards improved biological outcomes. In this study, we aimed at closing in on the effects of nanotubular $\mathrm{TiO}_{2}$ surfaces with variable nanotopography on the response on human mesenchymal stem cells (hMSCs). Although the influence of $\mathrm{TiO}_{2}$ nanotubes on the cellular response, and in particular on hMSC activity, has already been addressed in the past, previous studies overlooked critical morphological, structural and physical aspects that go beyond the simple nanotube diameter, such as spatial statistics.

Methods: To bridge this gap, we implemented an extensive characterization of nanotubular surfaces generated by anodization of titanium with a focus on spatial structural variables including eccentricity, nearest neighbour distance (NND) and Voronoi entropy, and associated them to the hMSC response. In addition, we assessed the biological potential of a twotiered honeycomb nanoarchitecture, which allowed the detection of combinatory effects that this hierarchical structure has on stem cells with respect to conventional nanotubular designs. We have combined experimental techniques, ranging from Scanning Electron (SEM) and Atomic Force (AFM) microscopy to Raman spectroscopy, with computational simulations to characterize and model nanotubular surfaces. We evaluated the cell response at $6 \mathrm{hrs}, 1$ and 2 days by fluorescence microscopy, as well as bone mineral deposition by Raman spectroscopy, demonstrating substrate-induced differential biological cueing at both the short- and long-term.

Results: Our work demonstrates that the nanotube diameter is not sufficient to comprehensively characterize nanotubular surfaces and equally important parameters, such as eccentricity and wall thickness, ought to be included since they all contribute to the overall spatial disorder which, in turn, dictates the overall bioactive potential. We have also demonstrated that nanotubular surfaces affect the quality of bone mineral deposited by differentiated stem cells. Lastly, we closed in on the integrated effects exerted by the superimposition of two dissimilar nanotubular arrays in the honeycomb architecture.

Discussion: This work delineates a novel approach for the characterization of $\mathrm{TiO}_{2}$ nanotubes which supports the incorporation of critical spatial structural aspects that have been overlooked in previous research. This is a crucial aspect to interpret cellular behaviour on nanotubular substrates. Consequently, we anticipate that this strategy will contribute to the unification of studies focused on the use of such powerful nanostructured surfaces not only for biomedical applications but also in other technology fields, such as catalysis.

Keywords: nanotubes, nanotopography, spatial statistics, stem cells, bone quality 


\section{Introduction}

Elucidating the mechanisms that control how cells sense and respond to biomaterials surfaces has been the focus of a large body of literature in the past three decades. ${ }^{1-7}$ Results from these studies have unequivocally established that physicochemical properties such as roughness, topography, surface chemistry, energy and stiffness, direct cell fate by affecting key phenomena including adhesion, proliferation, differentiation, gene and protein expression. ${ }^{8-11}$ Among the factors known to control cellular events, nano-topographical features play a pivotal role by exerting direct cueing on adherent cells. ${ }^{2,9,10,12-17}$ Based on this evidence, much effort has been invested in the design of synthetic nanostructured substrates to support the investigation of the interplay between nanotopographical cues and cellular functions., ${ }^{2} 10,12,13,15,18$ Among the panoply of techniques developed to nanostructure biomaterials, anodization has rapidly emerged as a simple but effective electrochemical treatment to create bioactive nanostructures composed of arrays of aligned nanotubes on titanium, the gold standard in medicine. ${ }^{18-21}$ The design of morphological parameters, mainly nanotube diameter, has been achieved through the modulation of experimental parameters (i.e., anodization voltage and time, composition of the electrolytic solution), permitting to rationally engineer nanotopographical features and assess their influence on cellular events. $^{22,23}$ This allowed the determination, for example, that the inductive differentiation of hematopoietic stem cells into osteoclasts, as well as the proliferation of primary human osteoblast and rat mesenchymal stem cells, are enhanced on $15 \mathrm{~nm}$ wide nanotubes. ${ }^{24,25}$ Conversely, nanotubes larger than $50 \mathrm{~nm}$ dramatically reduce rat mesenchymal stem cell activity and induced apoptotic programmed cell death. ${ }^{25}$ More recently, human adipose-derived stem cells showed a diameter-dependent proliferation and differentiation elicited by nanotubes within a $30-45 \mathrm{~nm}$ diameter range. ${ }^{26}$ Taken together, these studies indicate that smaller nanotube diameters provide beneficial effects on many stem cell functions when compared to larger nanotubes. However, the conclusive modelling of stem cell response to nanotubular surfaces is still a controversial subject as additional cellular processes critical in stem cell functions are favoured by larger nanotubes, thereby calling for further investigation to address these discrepancies. ${ }^{27-29}$ In particular: (i) diameters ranging from 70 to $100 \mathrm{~nm}$ elicit a tenfold increase in human mesenchymal stem cell (hMSC) elongation and differentiation into osteoblast-like cells, ${ }^{30}$ (ii) 100 -nm diameter nanotubes promote greater differentiation of mouse bone marrow mesenchymal stem cells (mMSCs) when compared to $30 \mathrm{~nm}$ tubes, ${ }^{31}$ (iii) 65-85 $\mathrm{nm}$ nanotubes provide the optimal substrate for the proliferation and osteogenic differentiation of human adiposederived stem cells (hADSCs) ${ }^{26,32}$ and (iv) mouse bone marrow stromal cells (BMSCs) responded to 100-nm nanotubes with increased osteogenic differentiation compared to $30-\mathrm{nm}$ nanotubes. ${ }^{33}$ Although these inconsistencies may be due to the specific attributes of different cell lines employed in these studies (e.g., source tissue, species, donor-donor variation), there is undoubtedly morphology-dependent cueing exerted by nanotubes on cellular processes that warrants a more stringent investigation.

Notably, while seminal work has focused on nanotube diameter as the sole independent variable to explain the cell response to nanotubular surfaces, several yet potentially equally important morphological (e.g., inner-tube eccentricity and inter-tube spacing) and spatial parameters (e.g., geometric arrangement) were overlooked. The latter is especially critical for human mesenchymal stem cells as symmetry and disorder in circular nanopit arrays have been shown to influence hMSC functions. ${ }^{34}$ In fact, it was shown that nanoscale disorder induces osteogenic differentiation and subsequent bone production in the absence of osteogenic supplements. Conversely, ordered nano-topographies limit cellular adhesion and osteoblastic differentiation. Accordingly, it could be conceived that prior contradicting results with nanotubular surfaces derive from lack of comprehensive characterization of nanostructures beyond the diameter and, more importantly, from the absence of stringent quantitative analysis of spatial statistics, given the principal role of symmetry and disorder. ${ }^{35}$ Therefore, a cohesive protocol which comprehensively includes morphological and spatial analyses for stem cellnanotube interactions is expected to provide the key to interpret cellular behaviour on anodized titanium and to unify the approach for the investigation of not only nanotubular surfaces, but also of any substrate characterized by arrays of circular/oval pits.

Anodization has also proven to be a versatile and inexpensive method to generate complex self-assembled structures with the precise control over the surface features through modulation of key parameters and procedures (e.g., multistage protocols), permitting the distinctive advantage of fabricating hierarchical architectures (e.g., double-walled structures, nanorods, and nanowires). ${ }^{19}$ Among these, morphologies such as lotus root-shaped/honeycomb structures ${ }^{36-39}$ present a unique opportunity for a paradigm shift in the biomedical field, where synthetic multiscale architectures are increasingly 
explored to generate complex 3D-environments and geometries to support and regulate cellular activity. ${ }^{40,41}$

In this work, we aimed at bridging the gap in previous literature by introducing a series of morphological and spatial analyses for the characterization of nanotubular architectures, culminating in the descriptive analysis of nanotubular surfaces towards a cohesive investigation of cell-nanotube interactions. To this end, we investigated three nanotubular arrays with variable nanotube diameters and a two-tiered honeycomb structure. Successively, we carried out morphological and spatial analysis (e.g., Voronoi entropy) to quantify the tubular geometry, arrangement as well as degree of order for these architectures. Our experimental data was validated by computational simulations to provide greater insight into the role of morphological parameters and spatial statistics. Subsequently, we evaluated the effects of the four surfaces on hMSC bioactivity (i.e., proliferative and morphological analyses) and osteogenic induction (e.g, commitment to osteogenic differentiation and bone mineral quality). Results from our study (i) highlight the importance of including additional morphological analyses and spatial statistics in the characterization of nanotubular surfaces for the purpose of enhancing the validity of cross-study comparisons, (ii) provide a comprehensive correlation between a multifactorial array of these parameters and hMSC activity extending from adhesion to bone mineral deposition, and lastly (iii) report the synergistic effects elicited by the $\mathrm{HC}$ architecture.

\section{Materials and Methods Nanotubular Arrays and Two-Tiered Honeycomb (HC) Structure}

Titanium foil $(0.127 \mathrm{~mm}$ thick, $99.9+\%$ purity, Alfa Aesar, USA) was cut into $2.5 \times 1 \mathrm{~cm}$ pieces, ultrasonically cleaned in toluene, rinsed with deionized (DI) water and finally dried in air. Samples were then subjected to a two-step anodization procedure in an electrolyte solution containing $0.3 \mathrm{wt} \%$ crystalline ammonium fluoride $\left(\mathrm{NH}_{4} \mathrm{~F}\right.$, Sigma Aldrich, USA) and $2 \mathrm{wt} \%$ DI water in anhydrous ethylene glycol $\left(\mathrm{C}_{2} \mathrm{H}_{6} \mathrm{O}_{2}\right.$, Sigma Aldrich). A two-electrode configuration was used to carry out the anodization treatments with a platinum foil counter electrode $(25 \times 25 \times 0.1 \mathrm{~mm}, 99.9 \%$, Alfa Aesar). A custom 3D-printed apparatus for reliable electrode alignment (Figure 1A) was designed in Fusion360 (Autodesk, USA) and printed with $1.75 \mathrm{~mm}$ PLA filament (AMZ3D, USA) on an I3 Mega $\mathrm{S}$ (ANYCUBIC, China). The apparatus was designed to fit atop of a $50 \mathrm{~mL}$ Pyrex beaker, containing exactly $30 \mathrm{~mL}$ of the electrolyte solution, and retained a stable distance between the electrodes of $2 \mathrm{~cm}$ to ensure consistent results, ${ }^{42}$ without contamination of the alligator clips by the solution. After the first step of anodization, samples were rinsed with DI water, dried in air and the oxide layer peeled off with tape to expose the newly formed nucleation sites for the subsequent generation of nanotubes during the second anodization step (Figure 1B). The formation of nucleation sites resulted in the fabrication of nanotubular substrates with a higher degree of order, compared to those obtained by onestep anodization. ${ }^{43}$ Samples were thoroughly rinsed in DI and dried prior to being cut into two pieces of $1 \times 1 \mathrm{~cm}$. Table 1 contains the applied voltages and times for the fabrication of nanotubular surfaces characterized with average diameters $\sim 20, \sim 50$ and $\sim 90 \mathrm{~nm}$ (hereafter referred to as NT1, NT2 and NT3, respectively) as well as the two-tiered honeycomb surface (hereafter referred to as HC) composed of arrays of smaller nanotubes (s-HC) clustered within larger domains (L-HC).

\section{Morphological Characterization}

The surface morphology of anodized substrates was investigated by using a Scanning Electron Microscope (6610LV SEM, JEOL, Japan) at 45,000X magnification. SEM images were successively analyzed by the image analysis software $\mathrm{Fiji}^{44}$ and by a custom CellProfiler pipeline $^{45}$ to quantify average nanotube diameter, eccentricity, wall thickness as well as nearest neighbour distance (NND).

The topography of the HC surfaces was characterized by a Dimension AFM instrument (Veeco Instruments, New York, USA) in tapping mode. We employed a rectangular $\mathrm{Si}_{3} \mathrm{~N}_{4}$ cantilever characterized by a nominal spring constant $(k)$ of $42 \mathrm{~N} \cdot \mathrm{m}^{-1}$, resonance frequency $(f)$ of $330 \mathrm{kHz}$ and a non-rotated pyramid tip with a nominal radius of $10 \mathrm{~nm}$ (PPP-NCHR, Nanosensors, Switzerland). Data were subsequently processed and analyzed in WSxM for the step-height along the $\mathrm{z}$-axis between the two nanotubular domains of the HC surface. ${ }^{46}$

\section{Spatial Analysis and Entropy Simulations}

SEM micrographs were subjected to a custom CellProfiler pipeline, involving the erosion of the nanotube apertures to their respective centroids, followed by 2D Voronoi tessellation to generate the experimental planar tiling for subsequent analysis. Surface Voronoi entropy $\left(S_{v}\right)$ was calculated as a metric of systemic order, defined by $S_{v}=-\sum_{n} P_{n} \ln P_{n}$, 


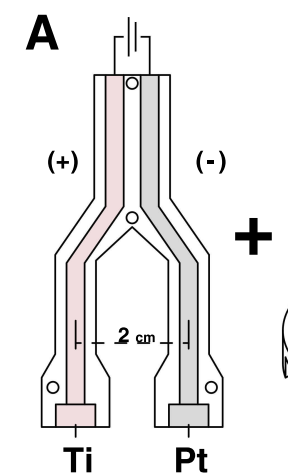

$\mathrm{Ti}$
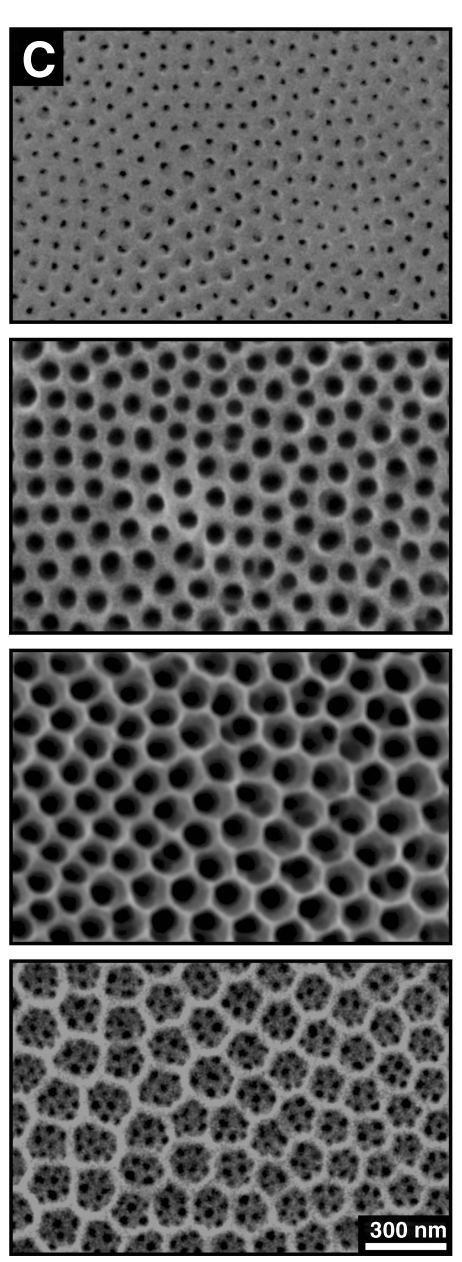
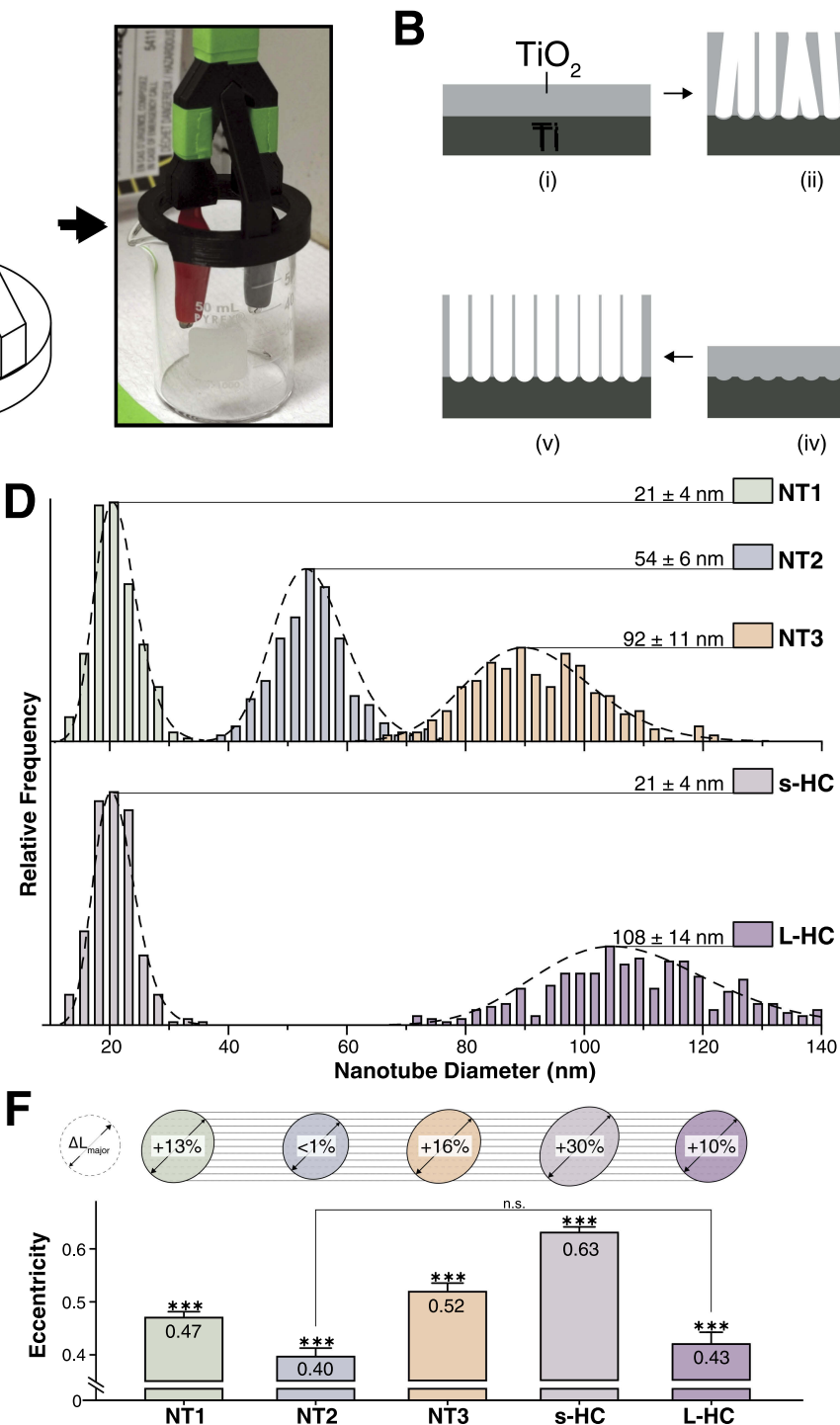

(ii)

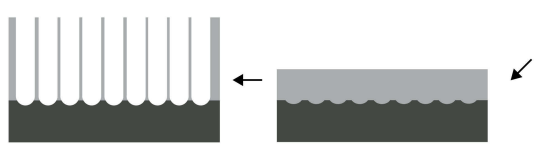

(iv)
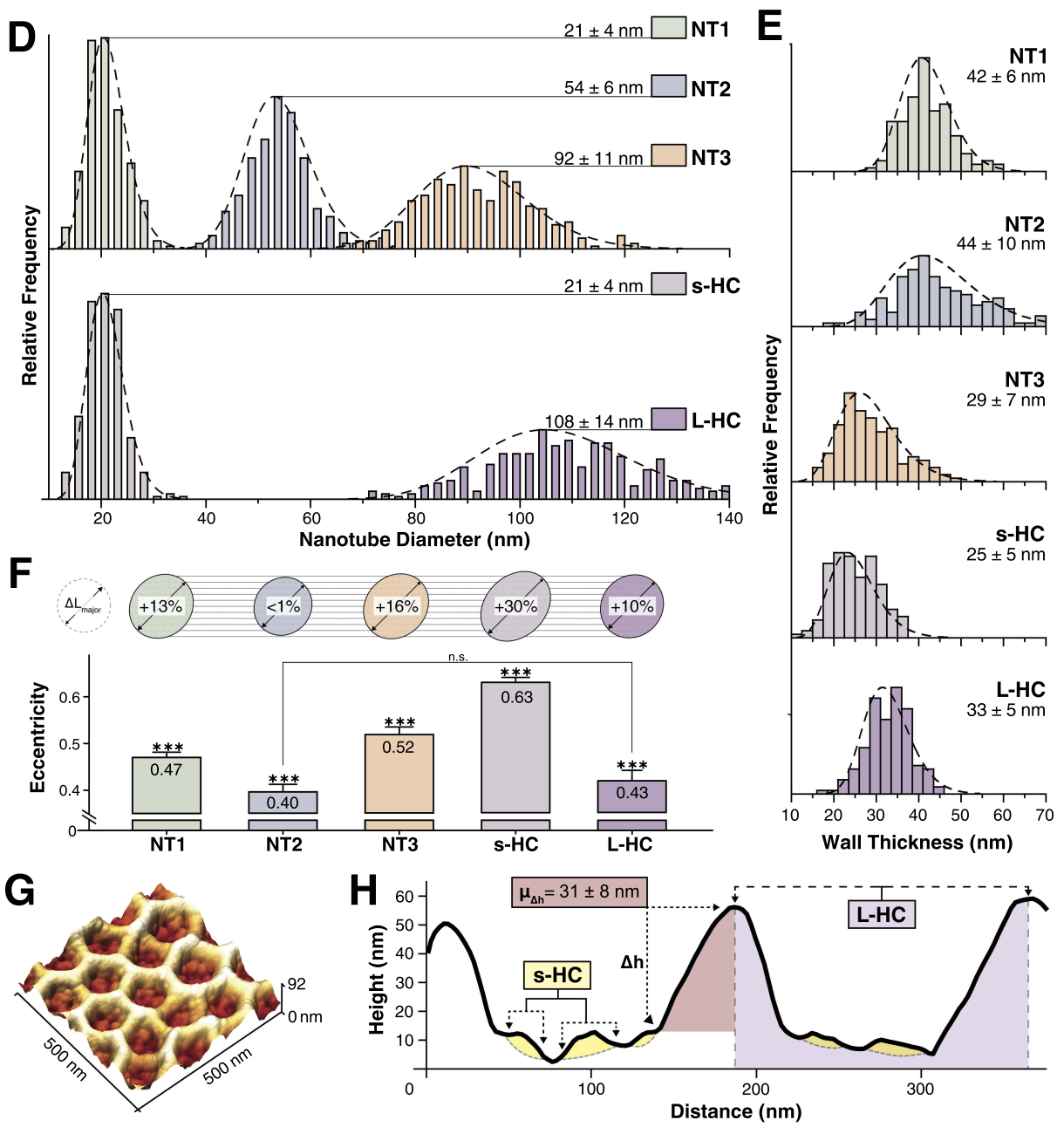

Figure I Nanotube generation, imaging and structural analyses. (A) Custom-made set up for anodization (B) Protocol of substrate generation via a two-step anodization process. (C) SEM images of the nanotubular surfaces categorized as NTI, NT2, NT3 and HC. (D) Diameter and (E) inter-tube wall thickness distributions. (F) Eccentricity analysis of nanotube morphology (bottom) with relative comparison across conditions (top). KW-ANOVA statistical comparison with $* * *=\mathrm{P}<0.00 \mathrm{I}$ against all other conditions. (G) 3D-AFM micrograph of the $\mathrm{HC}$ surface. $(\mathbf{H})$ Representative line profile derived from AFM images to quantify the vertical gap ( $\Delta \mathrm{h}$ ). Statistical significance was defined as $* * *=P<0.001$.

where $P_{n}$ is the fraction of polygons with $n$ sides. ${ }^{47,48}$ Data were recorded and processed in OriginPro software (OriginLab, USA).

Different experimentally observed effects have been reproduced in simulations with Mathematica (Wolfram,
USA) to investigate possible simple relations between the nanotubes' distributions, geometric patterns and the collective measure quantified by the entropy. Details are provided in the Supplementary Information and Figures S1-S8. 
Table I Experimental Parameters Used to Create Nanotubular Arrays (NTI, NT2 and NT3) and the Dual-Layer Honeycomb Structure $(\mathrm{HC})$

\begin{tabular}{|l|l|l|l|l|l|}
\hline & Condition & NTI & NT2 & NT3 & HC \\
\hline Step I & Voltage (V) & 30 & 40 & 60 & 60 \\
& Time (Min) & 60 & 45 & 30 & 30 \\
\hline Step 2 & Voltage (V) & 30 & 40 & 60 & 20 \\
& Time (Min) & 5 & 25 & 10 & 20 \\
\hline
\end{tabular}

\section{Contact Angle Measurements}

The static water contact angle was measured with a VCA Optima Surface Analysis System (AST Products Inc., USA). A $1 \mu \mathrm{L}$ drop of DI was deposited through a micro-syringe (Hamilton Company, USA) onto the substrates. Manual angular measurement of the droplet was performed to ensure accuracy prior to the automated measurement of the angle. Three droplets per sample were measured, and measurements were carried out on three samples per condition.

\section{Cell Cultures and Immunofluorescence Imaging \\ Bone \\ Marrow-Derived Human Mesenchymal Cells (hMSCs) Cultures}

Three different sources of bone-marrow-derived human mesenchymal stem cells (hMSCs) were obtained by two distributors (Lonza, USA and RoosterBio, USA) to account for donor variability. hMSCs from RoosterBio (Lot. 00014; Lot. 00082) were expanded in High-Performance Media (RoosterBio) and were thawed in similar media. hMSCs from Lonza (Lot. 603525) were expanded in Mesenchymal Stem Cell Growth Medium BulletKit (MSCGM, Lonza) and were thawed in similar media. Experiments were performed with low-passage hMSCs $(\leq \mathrm{P} 5)$ cultured in DMEM with 4.5 $\mathrm{g} \cdot \mathrm{L}^{-1}$ glucose and L-glutamine (Corning, USA), supplemented with $8 \%$ fetal bovine serum (FBS, Gibco, USA), 100 $\mathrm{U} \cdot \mathrm{mL}^{-1}$ penicillin and $100 \mu \mathrm{g} \cdot \mathrm{mL}^{-1}$ streptomycin (Gibco). The culture was maintained in a $5 \% \mathrm{CO}_{2}, 37{ }^{\circ} \mathrm{C}$ waterjacketed incubator. Cells were passaged with 1x TrypLE (Gibco) and suspension to be used for experiments were treated with $50 \mu \mathrm{g} \cdot \mathrm{mL}^{-1}$ Soybean Trypsin Inhibitor (SigmaAldrich) prior to seeding.

Nanotubular substrates were sterilized in $70 \%$ ethanol $(\mathrm{EtOH})$ and thoroughly washed in sterile $1 \mathrm{x}-\mathrm{PBS}$, prior to use in cell culture experiments. They were placed in 24-well plates and $500 \mu \mathrm{L}$ of the cell suspension was added to each well at a density of 10,000 cells $\cdot \mathrm{cm}^{-2}$. Cells for proliferation, morphology, focal adhesions (FAs) and bone mineral deposition studies were cultured on the nanotubular substrates for the experiment-specific time periods, ranging from 6 hours (h) to 28 days. Timepoints within the first $48 \mathrm{~h}$ were used for morphological analyses as these intervals provide insight into the initial cell-substrate effects, prior to differentiation-induced transformations. These experiments were terminated by cellular fixation with $500 \mu \mathrm{L}$ of fresh 4\%-paraformaldehyde (PFA, Sigma-Aldrich) for 10 minutes at room temperature. Analysis of mineral quality was assessed at 28 days ( $i$ ) for consistency with previous work, ${ }^{26,30,49}$ and (ii) to provide sufficient time for the mineral to be deposited and to undergo maturation (e.g., the substitution of carbonate in hydroxyapatite). ${ }^{50}$ These experiments were terminated by the removal of cell media and incubation within a desiccator to eliminate cell function while preserving the deposited minerals for spectroscopic analysis. All experiments were performed with 3 samples per condition, per time point, and were completed in triplicate.

For fluorescent imaging, cells were permeabilized with $0.25 \%$ Triton X-100 (TX-100, Sigma-Aldrich) for $10 \mathrm{~min}-$ utes at room temperature (RT) and labelled for nuclei (ReadyProbes NucBlue [DAPI], Thermo Fisher) and actin (Rhodamine Phalloidin, Thermo Fisher). To visualize focal adhesions, samples were blocked in $1 \%$-albumin/10\%normal goat serum (Thermo Fisher) for $1 \mathrm{~h}$ at RT. Vinculin was labelled with a primary 1:400 anti-hVin1 mouse $\mathrm{mAb}$ (Sigma-Aldrich) and secondary 1:800 AlexaFluor 488 goat-anti-mouse IgG (Thermo Fisher). Primary antibody was an overnight incubation while blocking and secondary antibody incubations were for $1 \mathrm{~h}$ each at RT. Samples were washed and mounted on \#1.5 VistaVision rectangular cover-glass slides (VWR, USA) with VECTASHIELD Vibrance (Vector Labs, USA) hard-set mounting media.

\section{Immunofluorescence Imaging}

Multi-channel images, used in the quantification of nuclei number and cytoskeletal morphological analysis, were captured with an AxioObserver.Z1 inverted microscope (Zeiss, Germany) fitted with an AxioCam MRm CCD (Zeiss). Nuclei were imaged using $5 \times 5$ tiles visualized through a 10x A-Plan objective ( $\mathrm{Ph} 2, \mathrm{NA}=0.25$, Zeiss). Cytoskeletal elements were observed using $5 \times 5$ z-stack tiles, visualized through a $20 \mathrm{x}$ Plan-Apo objective (Ph2, NA=0.8, Zeiss). Morphological analysis of the nuclei and focal adhesions was observed through at 40x PL APO Oil objective (NA=1.3, Leica) with a Quorum Spinning-disk Confocal setup (Quorum 
Technologies, Canada) affixed to a Leica BMI16000B inverted microscope (Leica Microsystems, Germany), complemented by a Photometrics Prime BSI sCMOS $[95 \%$ QE, back illuminated]. The setup was driven by MetaMorph software (Molecular Devices, USA).

Cell count was determined by quantifying the number of DAPI-stained nuclei on each surface with a custom CellProfiler pipeline. Cell count was then normalized against the average cell number per experiment, thereby generating the cell proliferation index (CPI). This was done to reduce inter-experiment variability. Nuclear and actin morphological analysis were achieved by stitching and focus-stacking the panels in Fiji ("Stack Focuser" package) ahead of their processing in a custom pipeline in CellProfiler. Key morphological parameters were the object area, eccentricity, form factor and protrusive index. The index of eccentricity $(e)$, an ellipse model of circularity, was measured for both nanotubes and cells. It is calculated by determining the ratio of the distance of the focal length of the ellipse and the major axis length. ${ }^{45}$ This entails that a perfectly circular object has $e=0$ and a line segment $e=1$. Form factor $\left(F_{f}\right)$, a measure of shape irregularity, was measured and is defined as $F_{f}=\frac{4 * \pi * A}{P^{2}}$ where $A$ is the area and $P$ is the perimeter. ${ }^{45}$ A perfectly round object has an $F_{f}=1$ whereas jagged contours yield $F_{f}<1$. For consistency with previous work, ${ }^{51}$ the form factor for the cellular and nuclear morphologies was labelled as the "cell shape index" (CSI) and "nuclear shape index" (NSI), respectively. The measure of "extent" was used as an indicator of protrusion, herein termed the "cell protrusive index" (CPI), and is calculated by the proportion of area in a surrounding bounding rectangle covered by the cell; where perfect coverage would be $=1$. In the case of elevated protrusiveness, the great number and length of anisotropic protrusions would widen the bounding box without shared isotropic spreading of the cell body, thereby lowering the CPI (i.e., elevated protrusion $=$ lower CPI). Images used for focal adhesion analysis were subjected to a custom macro in FijiImageJ that included focus stacking, background subtraction ("Rolling Ball Background Subtraction") and contrast enhancement ("Contrast Limited AHE", CLAHE) packages. Peripheral focal adhesion length was then manually measured in FIJI-ImageJ. All resultant data was compiled and processed in GraphPad Prism (GraphPad Software, Inc.) or OriginPro.

\section{Raman Spectroscopy}

Raman spectroscopy was employed to characterize the crystallinity of the nanotubular layers as well as the physicochemical properties of bone mineral deposited by hMSCs after 4 weeks of culture. To this end, a WITec Alpha 300 Raman microscope was used. The crystallinity was determined by focusing a $532 \mathrm{~nm}$ frequency-doubled Nd:YAG laser through a 20x EC EpiPlan objective (NA=0.4, Zeiss) on the surface of the nanotubular substrates, with a 30 -second acquisition time. The assignment of Raman bands for anatase and rutile titania peaks was carried out in accordance with previous literature. ${ }^{52}$

For the analysis of bone mineral, substrates were collected after 28 days of culture directly from the 24-well culture plate and gently rinsed in DI water to remove the excess of culture media prior to Raman measurements. Successively, Raman spectra were acquired by using a 100x EC EpiPlan NEOFLUAR objective $(\mathrm{NA}=0.9$, Zeiss) and a $785 \mathrm{~nm}$ diode laser with a 240 -second integration time and double acquisition to enhance the signalto-noise ratio. Three different substrates per condition were used, and experiments were carried out by analyzing samples that were derived from two independent cultures. Randomly selected mineral nodules were analyzed in situ, directly on each substrate to minimize perturbations associated with the collection of organic/inorganic material from the substrates. The number of mineral nodules analyzed per condition was dictated by the ease at which these could be identified on the substrates by optical microscopy and by the quality of the resulting Raman signature to ensure accurate peak deconvolution. Raman data were compiled and processed in OriginPro.

The principal Raman mineral bands used for quantitative analysis include the phosphate $\left(\mathrm{PO}_{4}{ }^{3-}\right) v 1$ band at $\sim 960 \mathrm{~cm}^{-1}$ and the B-type carbonate $\left(\mathrm{CO}_{3}{ }^{2-}\right) v 1$ band at $\sim 1070 \mathrm{~cm}^{-1} \cdot{ }^{53-56}$ The position and intensity of these bands provide information about mineral parameters such as crystal structure and deviations from stoichiometry. The most relevant Raman collagen bands to assess bone mineral quality are the amide I at $\sim 1660$ $1680 \mathrm{~cm}^{-1}$, the hydroxyproline bands at $855 \mathrm{~cm}^{-1}$ and $875 \mathrm{~cm}^{-1}$ and the amide III at $1245-1270 \mathrm{~cm}^{-1}$. The position, intensity, area and width of these bands are sensitive to changes in protein structure. ${ }^{57,58}$ In this study, the surface-induced alterations of the bone mineral were investigated using three metrics: mineralto-matrix ratio, carbonate-to-phosphate ratio and 
crystallinity. The analysis of spectral data requires quantification of peak parameters, such as position, the inverse of full-width-at-half-maximum (1/FWHM) and peak area. This was achieved by fitting the spectra with Lorentzian functions after background subtraction. Mineral-to-matrix ratio, a measure of the mineral content in bone, was calculated as the ratio of the integrated area of the bands located at 900-1000 and 1590-1720 $\mathrm{cm}^{-1}$. Carbonate-to-phosphate ratio, which denotes the carbonate content of the mineral in the bone specimen, was calculated as the intensity ratio of the carbonate $v 1 \quad\left(1070 \mathrm{~cm}^{-1}\right)$ to phosphate $v 1$ $\left(960 \mathrm{~cm}^{-1}\right)$ peaks. Mineral crystallinity, which depends on the disorder in the bone structure, was calculated from the 1/FWHM of the phosphate $v 1$ band $\left(960 \mathrm{~cm}^{-1}\right) .{ }^{59-62}$

\section{Statistical Analysis}

All experiments in this work were repeated in triplicate with at least three samples/condition. Bar charts are expressed as the mean with a $95 \%$ confidence interval (CI). Data sets were tested for normality using the Shapiro-Wilk test. Normal data sets were tested with the parametric Two-Way ANOVA with Tukey's HSD post-hoc test for mean comparisons. A difference was considered significant if $\mathrm{P}<0.05$. Statistical significance was defined as $*=\mathrm{P}<0.05, * *=\mathrm{P}<0.01$ and $* * *=\mathrm{P}<0.001$. A comparison of distributions was completed using the non-parametric Kolmogorov-Smirnov (K-S) statistical test, ${ }^{63}$ where the deviations between cumulative fraction functions are compared. Quantitative Raman data, which were confirmed for normality, were subsequently tested with Welch's $t$-test which is the recommended statistical method due to the unequal variances (confirmed with Bartlett's test) and unequal sample sizes. ${ }^{64}$ Statistical significance was defined $*=\mathrm{P}<0.05, \quad * *=\mathrm{P}<0.01$ and $* * *=\mathrm{P}<0.001$. Analyses were performed with GraphPad Prism and OriginPro.

\section{Results and Discussion \\ Surface Characterization NT Morphological Analysis}

We have capitalized on the distinctive potential of anodization to engender self-ordered nanotubular substrates for the investigation of the interplay between differently sized nanotubes and bone-derived human mesenchymal stem cells. Specifically, a two-step anodization protocol was employed to generate arrays of titania nanotubes, and rational variations in treatment parameters (i.e., time and voltage; Table 1) allowed us to modulate the resulting morphology (Figure 1C). These surfaces were generated with matched voltages between each anodization step to enable the growth of aligned nanotubes with superior order from well-defined nucleation sites formed during the first stage. ${ }^{37,39}$ In an effort to investigate, for the first time, synergistic/antagonistic effects elicited by hierarchical nanotubular structures, we engineered a two-tiered surface characterized by clusters of smaller nanotube assemblies confined within larger domains. We first generated the first nanotubular layer with a voltage associated with the NT3 condition $(60 \mathrm{~V})$, with the intention to generate discrete regions for concentrated and clustered nanostructure growth in the subsequent stage. After the removal of the first stage oxide layer, which exposed the newly formed nucleation sites, the second stage nanotubular layer was generated with a voltage associated with the NT1 condition $(20 \mathrm{~V})$. The resulting two-tiered honeycomb architecture (HC) was successfully imaged by SEM (Figure 1C) and was found to be comprised of clusters of 5-7 smaller nanotubes (s-HC) contained within larger domains (L-HC).

Image analysis of the SEM micrographs confirmed the expected relationship between the applied voltage and nanotubes' lumen diameters, ${ }^{37}$ with voltages of 20,40 and $60 \mathrm{~V}$ resulting in mean diameters of 21, 54 and $92 \mathrm{~nm}$ (labelled as NT1, NT2 and NT3, respectively). A descriptive evaluation of the $\mathrm{HC}$ architecture established that s-HC domains (mean diameter of $21 \mathrm{~nm}$ ) are enclosed within larger L-HC boundaries (mean diameter of $108 \mathrm{~nm}$ ). Therefore, while the s-HC nanotube array is identical, diameter-wise, to the NT1 surface, the L-HC was significantly larger than the NT3 nanotubes. In order to quantify these observations, we carried out the Kolmogorov-Smirnov (K-S) testing on the diameter distributions (Figure 1D) and confirmed both the observed difference between the NT3 and L-HC domains (K-S, P $<0.001)$ and the similarity between NT1 and s-HC domains (K-S, P=0.5842). This allowed us to maintain consistency for at least one morphological variable for the comparison between the NT1 surface and the $\mathrm{HC}$ architecture. This was particularly critical for the interpretation of cell behaviour the $\mathrm{HC}$ architecture. Ultimately, this has permitted us to single out variations in cellular response exclusively induced by other variables, including order and clustering of these $\sim 20 \mathrm{~nm}$ features as well as the presence of the overlaying L-HC array. 


\section{Spatial Organization of NTs}

To complete the morphological characterization, we quantified the inter-tube spacing which, due to the continuous nature of our surfaces, can thereby be referred to as the shared wall widths between the nanotubes. This is opposed to common free-standing nanotubes where a gap, which is often disregarded, exists between the discrete walls of neighbouring tubes. Distributions of these widths are shown in Figure 1E. NT1 and NT2 displayed mean values of $42-44 \mathrm{~nm}$, while NT3 was characterized by significantly narrower walls at $\sim 29 \mathrm{~nm}$. In this context, Biggs et al. indicated that focal adhesion formation occurs in the interpit region. ${ }^{34}$ In addition, it was shown that integrin clustering and the subsequent cellular adhesion improve as the interpit separation increases, identifying optimal effects for $<70 \mathrm{~nm} .{ }^{65}$ This work conforms with our working hypothesis that, at the nanoscale, the order of interpit features (i.e., spacing) play an equally important role in the control of cellular adhesion and function. Further, introducing disorder and/or increasing the interpit spacing is suggested to facilitate focal adhesion formation which would then positively affect the process of cellular spreading. ${ }^{65}$ Notably, similar optimal spacing values were found to apply to integrin-specific ligand nanopatterns. ${ }^{66}$ Translated to our study, as our wall thicknesses are $\sim 43 \mathrm{~nm}$, we expect integrin clustering, and thus focal adhesion formation, to occur readily on NT1 and NT2. However, as the intratube diameter on NT3 exceeds $70 \mathrm{~nm}(\sim 92 \mathrm{~nm})$, coupled with the thinnest walls $(\sim 29 \mathrm{~nm})$, thus the lowest interpit area among the single-level architectures, and so we expect it to show the most detrimental effects on cell adhesion and spreading. In the case of the $\mathrm{HC}$ architecture, the available surface for FA establishment may be regarded as the sum between the walls of the L-HC layer and those pertaining to the s-HC arrays.

The morphological characterization of anodized surfaces was completed by assessing the eccentricity of nanotubes (Figure 1F), a parameter that assesses circular morphology which has often been overlooked in the study of cell-nanotube interactions. Differences between conditions were confirmed by the nonparametric Kruskal-Wallis ANOVA test, due to the lack of normality in the data. For all NT surfaces, the nanotubes lumen displayed an elliptical outline (i.e., $e>0$ ) and was more marked in the NT3 surface. Conversely, the morphology closest to a perfect circle was displayed by NT2 substrate and L-HC domains. Notably, the s-HC condition showed the highest eccentricity, 30\% greater than the one measured on the NT2 surface.

\section{Additional HC Nanotube Characteristics}

Given the 3D nature of the $\mathrm{HC}$ architecture, AFM analysis was necessary to determine the height difference between the outmost surface of the inner s-HC tubes and the outer surface of the L-HC domains (Figure 1G). This revealed a vertical step height difference $\left(\Delta \mathrm{h}_{\mathrm{HC}}\right)$ of $\sim 31 \mathrm{~nm}$ (Figure $1 \mathrm{H}$ ), crucial to the interpretation of our cellular results. In this context, it was shown that cells ( $\mathrm{C} 2 \mathrm{C} 12$ myoblasts) can sense, through integrins, adhesion proteins immobilized down to 100 -nm-deep nanopits. ${ }^{67}$ Considering the smaller vertical gap, we could thus expect that cells will be able to simultaneously sense both the L-HC and s-HC, thereby experiencing unique integrated cueing made possible by such two-tiered nanoarchitecture. Of note, depending on the point of reference, the L-HC array could be seen either as a planar surface characterized by arrays of $\sim 30$-nm-deep pits (point of reference: the outer surface of the L-HC) or as an array of $\sim 30$-nm-high protrusions overlaying the s-HC layer (point of reference: the s-HC layer). In this context, a review by Biggs et al. analyzed previous literature on cellular interactions with arrays and nanopits and nanoprotrusions, concluding that: ${ }^{65}$ (i) pit diameters greater than $70 \mathrm{~nm}$ perturb integrin clustering only when the $z$ dimensions of the pits exceed $\sim 100 \mathrm{~nm}$, (ii) cellular adhesion is decreased on structures measuring $\sim 70-100 \mathrm{~nm}$ in height and (iii) nanoprotrusion heights lower than $70 \mathrm{~nm}$ are insufficient to disrupt integrin formation and reinforcement (and, as a matter of fact, nanofeatures lower than $50 \mathrm{~nm}$ are comparable to planar substrates in terms of FA formation). Taken together, we can thus infer that, in either case, the vertical dimension of the L-HC layer is not expected to have any direct effect on FA clustering and, in turn, on the subsequent cellular spreading on the $\mathrm{HC}$ architecture.

\section{Spatial Analyses (Experimental)}

After morphological characterization, we closed in on the spatial statistics in order to obtain information about the geometrical arrangement and spatial proximity between nanotubes. To this end, we first considered the nearest neighbour distance (NND) and assessed the average distance between the centroid individual nanotubes and that of their closest neighbours. This parameter complements the morphological description of nanotubular structures, and it results from the combination of the spatial distribution of the nucleation sites created during the first anodization step, the nanotube diameter 

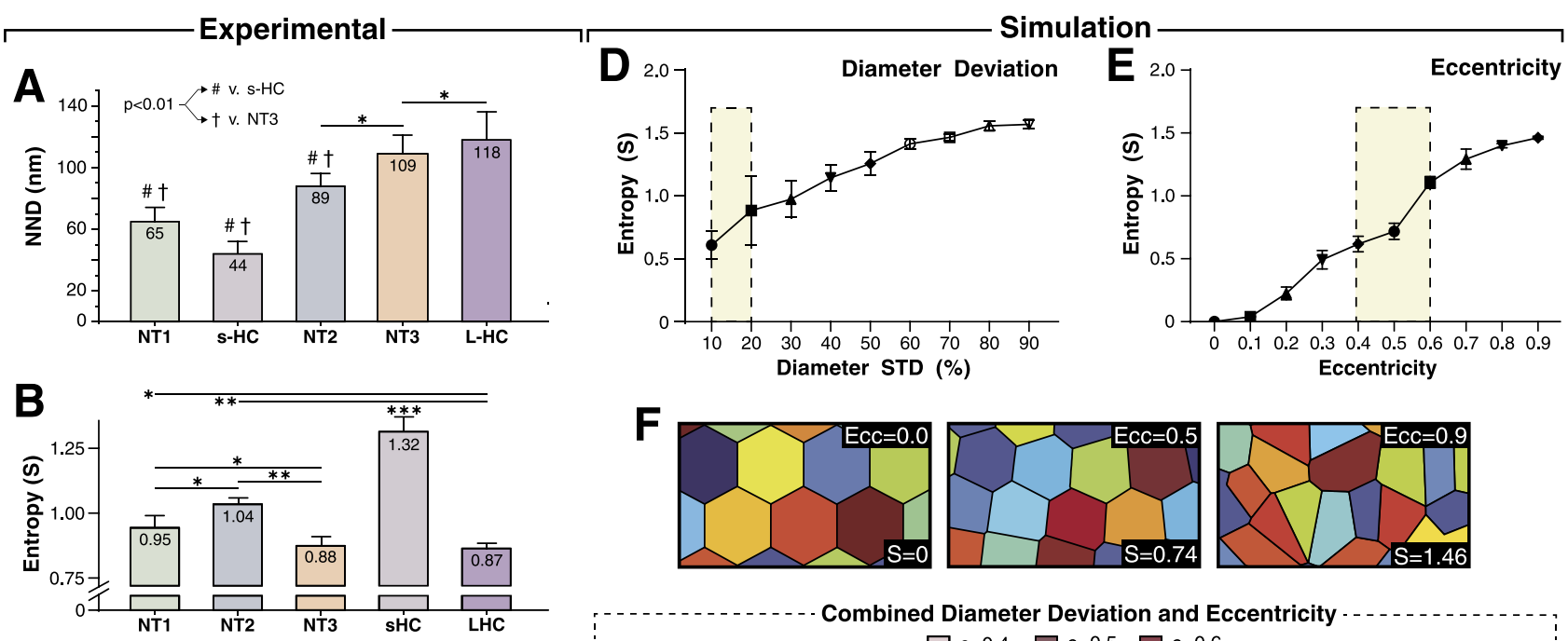

\begin{tabular}{|l|c|c|c|c|c|}
\cline { 2 - 6 } \multicolumn{1}{c|}{} & NT1 & NT2 & NT3 & s-HC & L-HC \\
\hline Diameter (Ø) & $\begin{array}{c}21 \\
\mathrm{~nm}\end{array}$ & 54 & 92 & 21 & 108 \\
\hline $\begin{array}{c}\text { Wall } \\
\text { Thickness }\end{array}$ & $\begin{array}{c}42 \\
\mathrm{~nm}\end{array}$ & 44 & 29 & 25 & 33 \\
\hline Eccentricity & 0.47 & 0.40 & 0.52 & 0.63 & 0.43 \\
\hline NND & $\begin{array}{c}65 \\
\mathrm{~nm}\end{array}$ & 89 & 109 & 44 & 118 \\
\hline Entropy (S) & 0.95 & 1.04 & 0.88 & 1.32 & 0.87 \\
\hline
\end{tabular}

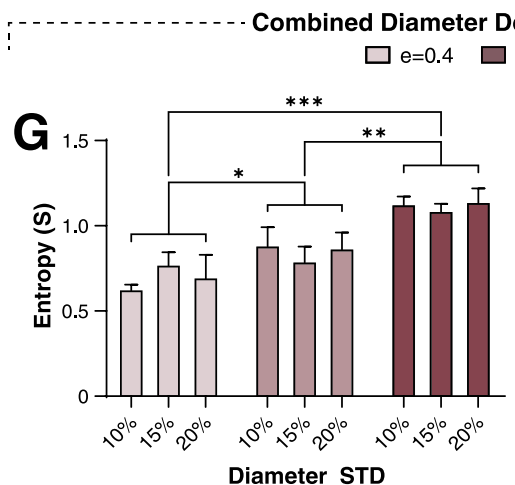

ation and Ecce

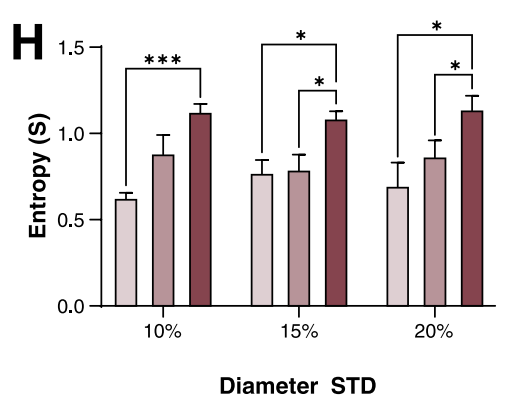

Figure 2 Spatial statistics analysis of nanotubular surfaces. (A) Experimental nearest neighbour distance (NND) and (B) Experimental Voronoi entropy. (C) Summary table of experimental data, showing (i) mean values of morphological parameters and (ii) ranking-based shading to visually compare properties across the different conditions tested. $20 \%, 40 \%, 60 \%, 80 \%$ and 100\% shading were used to rank morphological properties in ascending order based on their numerical values. Simulated effects of variations in (D) diameter and $(\mathbf{E})$ eccentricity on entropy. $(\mathbf{F})$ Examples of Voronoi tessellation on simulated ordered, semi-ordered and random patterns with entropy $\left(S_{v}\right)$ equal to $0,0.74$ and I.46, respectively. $(\mathbf{G}-\mathbf{H})$ Simulated combined effects of diameter and eccentricity deviation. Statistical significance was defined as $*=\mathrm{P}<0.05, * *=\mathrm{P}<0.0 \mathrm{I}$ and $* * *=\mathrm{P}<0.00 \mathrm{I}$.

and wall thickness. Figure 2A displays the NNDs for all conditions, showing the $\mathrm{HC}$ structure simultaneously comprises the smallest (s-HC array) and highest (L-HC array) NNDs. Notably, distances increased according to the diameter (e.g., $\mathrm{NND}_{\mathrm{NT1}}=65 \mathrm{~nm}, \mathrm{NND}_{\mathrm{NT3}}=110 \mathrm{~nm}$ ), which is consistent with the growth mechanism by which, as the nanotubes become larger, the centroids will diverge. We expanded our analysis to spatial statistics by generating Voronoi tessellations from the centroids of respective tubes, which allowed the subsequent assessment of surface entropy. Specifically, the Voronoi entropy was the lowest for the NT3 surface, indicating a more ordered arrangement when compared to the other NT conditions (Figure 2B); consistent with more marked constraining effects dictated by the larger size which limit their freedom to rearrange. In addition, the $\mathrm{HC}$ architecture displayed the highest values for the s-HC domains, denoting the smallest degree of order among all conditions analyzed. Such nanoscale semi-order is partly dictated by the spatial configuration of the initial nucleation sites created during the first anodization step, which affects the subsequent formation and growth of the nanotubes during the second step. ${ }^{37-39}$ In addition, the degree of order intertwines with other morphological variables such as nanotube diameter, wall thickness, eccentricity and NND. A 2-way relationship between the degree of order and the morphological parameters can, in fact, be observed (Figure 2C).

\section{Spatial Analyses (Simulations)}

To demonstrate this hypothesis, we simulated nanotubular patterns with variable degrees of order which were subjected to the same Voronoi tessellation methods and analyses applied to the experimental surfaces. Our intention was to identify the individual and combinatory effects of these parameters, namely diameter and eccentricity, with respect to the resultant entropy which would then be drawn on to explain the experimental cellular response. As expected, our simulations demonstrated that the entropy increases as the variability of the diameter and/ or of the eccentricity of elliptical nanotube-like features increase (Figure 2D and E), establishing a theoretical foundation to 

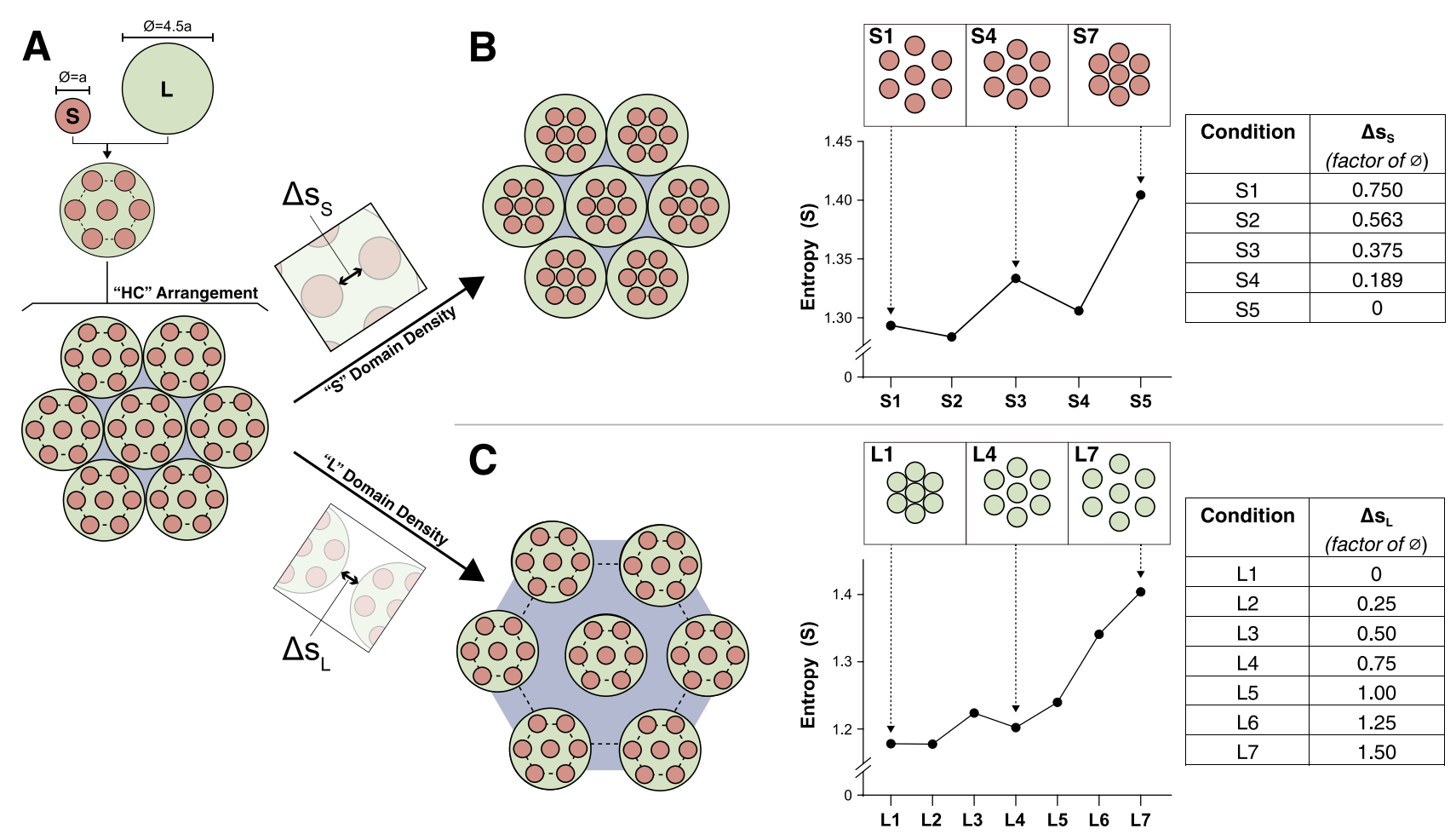

Figure 3 Simulated effects of domain spacing on entropy. (A) The setup is followed by simulation results from (B) tightening of small domain spacing and (C) expansion of large domain spacing.

explain the observed relationship between nanotubular morphology and disorder. Examples of Voronoi tessellations from the simulated conditions are shown in Figure 2F. While these simulations spanned across a large range of variability $(10-90 \%$ for diameter, $0-0.9$ for eccentricity), the variations of these parameters were relatively confined with respect to our experimental data (10-20\% for diameter, $0.4-0.6$ for eccentricity), and are identified as the yellow fields within Figures 2D and E. It can be seen that within these intervals, the entropy values are lower than those quantified experimentally (Figure 2C). This is likely due to the fact that on NT and HC surfaces, the individual contributions of variations in diameter and eccentricity are compounded. To quantitatively demonstrate this aspect, we simulated the combined effects of variations in diameter and eccentricity within the ranges set out by our experimental data (Figure 2G-H). Our results show that while variations in diameter do not significantly affect the entropy, even small variations in eccentricity (i.e., 0.4-0.6) cause drastic changes in entropy. This finding cements the importance of including tubular eccentricity as a major contributor to the overall degree of order in the characterization of nanotubular arrays.

\section{Analysis of the HC Nanoarchitecture}

Notably, the higher disorder determined for the s-HC nanotubes may also derive from the clustering dictated by $(i)$ the relative spatial arrangement of smaller tubes and/or (ii) the spatial constraints exerted by the larger L-HC domains. Computational simulations show in fact that by $(i)$ decreasing the relative distance among s-HC tubes and/or (ii) increasing the confinement action by expanding the spacing of the L-HC domains, while keeping the arrangement of the s-HC tube constant, the entropy increases (Figure 3). It can thus be postulated that by rationally modulating the voltage of the two anodization steps, the spatial arrangement of the nucleation sites and/or the wall thickness of the L-HC domains could be controlled, thereby offering a strategy to design the degree of disorder of the s-HC clusters and thus a way to optimize direct surface cueing on adhering cells.

\section{Analysis of the $\mathrm{TiO}_{2}$ Layer}

In addition to morphological analysis and spatial statistics, we applied Raman spectroscopy to assess the crystallinity of the nanotubular layers, a physical characteristic that plays an equally important role in controlling the response of adhering cells. ${ }^{6,69}$ Although anodization is expected to produce mostly amorphous $\mathrm{TiO}_{2}$ without post-annealing treatment, modification of time and water content in the electrolyte solution can nonetheless induce phase transformation to crystalline forms of titania (i.e., anatase, rutile). ${ }^{70}$ As the crystal structure of the oxide layer has been shown to 


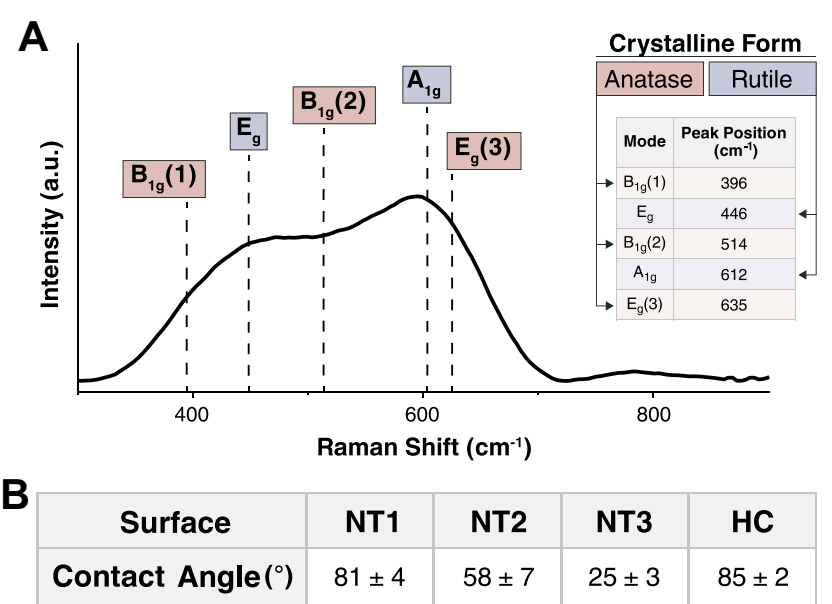

Figure 4 (A) Representative Raman spectrum on nanotubular architectures and Raman peak assignment. (B) Contact angle values.

exert direct effects on cellular activity, ${ }^{68,69}$ the degree of crystallinity of the nanotubular layer should be routinely assessed to standardize the evaluation of nanotubecontrolled cellular events. ${ }^{71}$ In this context, all surfaces investigated in this study displayed the distinctive spectrum of amorphous titania (Figure 4A), characterized by a broad band and by the absence of sharp peaks associated with the two crystalline phases (i.e., anatase and rutile). ${ }^{72}$ This confirms that anodization did not alter the amorphous nature of the native $\mathrm{TiO}_{2}$ layer.

Lastly, contact angle measurements assessed the wettability of surfaces (Figure 4B). Our data indicate that the contact angle decreased as the diameter of the NT substrates increased. Interestingly, the $\mathrm{HC}$ architecture displayed a contact angle value similar to that of the NT1 surface, suggesting that wettability is controlled by the s-HC layer given the similarities between these two conditions.

\section{Cellular Results Proliferation}

We successively evaluated the hMSC cell number at two different intervals to investigate topography-dependent effects on differential cellular proliferation. Figure 5A displays the cell number at 1 and 2 days of a culture determined by quantifying the number of DAPI-stained nuclei on each surface. At 24 h, NT surfaces displayed differences in cell counts, indicating a substrate-induced effect on cell growth exerted by nanotubes as reported in previous studies. In our case, the stark similarity between entropy (Figure 2B), the $24 \mathrm{~h}$ proliferation data (Figure 5A) and, as a matter of fact, early cell spreading data within the first $6 \mathrm{~h}$ (Figure 6A), suggests that the degree of disorder may play a key role at short intervals. At $48 \mathrm{~h}$, while cell count was statistically similar on the three NT surfaces, the HC architecture promoted cell growth, suggesting synergistic compounded effects since cells behave differently than on the respective nanotubular component counterparts (i.e., namely NT1 and NT3). Notably, we can ascribe the variations in CPI, especially significant for the NT1 and $\mathrm{HC}$ conditions, to their size distribution. Previous work has in fact shown that in the $15-20 \mathrm{~nm}$ diameter range, variations as small as $5 \mathrm{~nm}$ can yield a reduction of about $33 \%$ in cell proliferation. ${ }^{24}$ In our case, NT1 and s-HC nanotube diameter varied within such a critical interval (i.e., from $17 \mathrm{~nm}$ to $25 \mathrm{~nm}$ ) (Figure 1D) a factor that may have caused cells to experience compounded differential cueing resulting in a less homogenous overall behaviour. In addition, the NT3 substrate exhibited the lowest NSI (Figure 5B-C), thereby indicating a more marked nuclear deformation. This is most likely a result of cytoskeletal disorganization leading to the loss of structural integrity. ${ }^{73}$ On NT3, cells may be in fact unable to generate large/reinforced focal adhesions leading to a more disorganized actin cytoskeleton which, in turn, hinders its ability to resist deformation from both internal and external forces.

\section{Morphology}

We successively closed in on substrate-induced variations in cell morphology observed in immunofluorescence microscopy by quantifying cellular area, eccentricity, form factor (i.e., cellular shape) and protrusive index (Figure 6A-D). At 6 h, cells on the $\mathrm{HC}$ architecture displayed a marked spreading compared to those on NT substrates. In addition, eccentricity and reduced form factor similarly characterized cells adhering to NT1 and $\mathrm{HC}$ substrates. However, the $\mathrm{HC}$ elicited a significantly more protrusive morphology $(\mathrm{P}<0.01)$. This suggests that while cells on the NT1 and HC surfaces display a more advanced spreading stage with respect to NT2 and NT3, characterized by the more elongated and protrusive cellular morphology, the $\mathrm{HC}$ architecture yielded greater cell spreading and abundance/ length of protrusions. At $24 \mathrm{~h}$, cells on the NT1 and HC surfaces displayed the highest cell area, indicating comparable cueing most likely due to the similarity between the NT1 and the s-HC nanotubes. At this interval, increasing the NT diameter yielded a reduction in the cell area, as previously reported for MC3T3 osteoblastic cells. ${ }^{49}$ The same trend was also observed for the eccentricity: the $\mathrm{HC}$ showed the highest value, which gradually decreased from NT1 to NT3 surface (Figure 6B). The opposite tendency was observed for the CSI (Figure 6C). At this time 

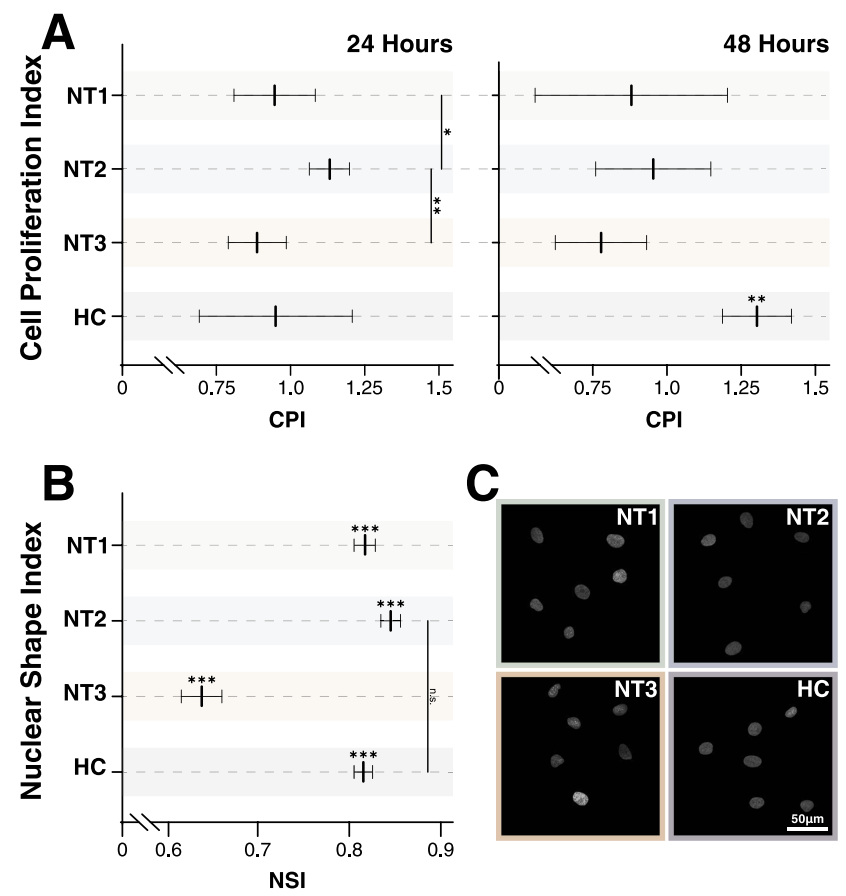

Figure 5 (A) Cell proliferation index [CPI] of hMSCs on nanotubular surfaces at $24 \mathrm{~h}$ and $48 \mathrm{~h}$. (B) Nuclear shape index [NSI] at $24 \mathrm{~h}$ and (C) representative images of hMSC nuclei at $24 \mathrm{~h}$. Statistical significance was defined as $*=\mathrm{P}<0.05$, $* *=\mathrm{P}<0.0 \mathrm{I}$ and $* * *=\mathrm{P}<0.00 \mathrm{I}$.

point, NT1 is the condition that elicits the most protrusive morphology $(\sim 0.485)$, followed by HC/NT2 $(\sim 0.525)$, suggesting that cells on the HC substrate are more spread and into migratory behaviour. At $48 \mathrm{~h}$, the trend observed in the cell area at one day was consolidated. However, the following changes occurred: (i) eccentricity of cells on NT1 matched that on HC substrates, (ii) cells on NT2 substrates showed the smallest relative value and (iii) a reversed trend in the form factor was found for the HC substrates. In addition, cells on the HC/NT1 surfaces display the most protrusive morphology, with the lowest protrusion index of any timepoint $(\sim 0.425)$. These morphological results can be summarized as follows: (i) cells on NT1/ HC surfaces exhibit the highest spreading, in terms of cell area, when compared to the other conditions; (ii) while cells on NT1 maintain a protrusive morphology, cells on the $\mathrm{HC}$ surface appear to advance from protrusive $(6 \mathrm{~h})$ to elongated $(24 \mathrm{~h})$ and multipolar (48 h) morphology as evidenced by considering the combined changes in eccentricity, form factor and protrusive index; (iii) cells on the NT3 surface (i.e., condition with the largest diameter and lowest entropy) exhibit the smallest surface area and a spherical morphology; (iv) cells on NT2 display intermediate features between such extremes. Taken together, our findings confirm the beneficial effects of topographies characterized by a combination of smaller diameters and higher entropy while demonstrating enhanced effects by the HC. This result is most likely associated with the greater disorder deriving from the higher eccentricity and clustering of the s-HC tubes, given that the L-HC array is not believed to exert direct cueing on FA clustering and cellular spreading because of its shallow nature. However, it may contribute to providing an extended surface for the establishment of FAs which makes up for the narrow wall thickness of the s-HC layer.

\section{Focal Adhesions}

To link the morphological analyses to the intracellular response, we focused on focal adhesions (FAs), the multimeric clusters of adhesion molecules which are crucial assemblies in mechanobiology that link components of the extracellular matrix (ECM) to intracellular structures (i.e., cytoskeletal elements). ${ }^{74}$ Importance of proper FA development and functioning of its constituents (e.g., focal adhesion kinase [FAK]) have been linked to several critical cell processes including migration, cell cycle progression and stem cell differentiation. ${ }^{75,76}$ Subsequently, FAs are able to be used as direct and indirect indicators of cell-substrate interaction and so a concerted effort has been seen in the analyses of these structures with static (e.g., size) and dynamic (e.g., turnover kinetics) measures. ${ }^{77-79}$ Of note, FA size have been indicated in key cell attributes including the prediction of cell migration, ${ }^{80}$ mechanoresponse, ${ }^{81}$ and stem cell biology. ${ }^{75,77}$ In this context, we measured the major axis length of the FAs at $24 \mathrm{~h}$ using immunofluorescent labelling of Vinculin (Figure $6 \mathrm{~F}$ ) - one member of the multimeric complex that has even distribution across the $\mathrm{FA}^{82}$ and acts as a key regulator of $\mathrm{FA}$ development as an adaptor protein to Talin and Actin. ${ }^{83,84}$ To this end, size distributions were determined (Figure 6E) and, in a similar fashion to nanotube morphological distributions, comparisons were completed using the $\mathrm{K}-\mathrm{S}$ statistical test. hMSCs seeded on NT2 $\left(\bar{\ell}_{N T 2}=3.65 \mu \mathrm{m}\right)$ and NT3 $\left(\bar{\ell}_{N T 3}=2.53\right.$ $\mu \mathrm{m})$ surfaces presented with FAs that were significantly smaller than on NT1 $\left(\bar{\ell}_{N T 1}=4.51 \mu \mathrm{m}, \mathrm{P}<0.001\right)$. These results coincide with previous literature that noted a marked decrease in focal adhesions on nanotubes with diameters approaching 100 $\mathrm{nm}$ (i.e., NT3), ${ }^{24,85}$ and optimal spacing for normal FA development in the $58-72 \mathrm{~nm}$ range, ${ }^{17,25}$ as seen with NT2 and NT3 surfaces. Notably, hMSCs seeded on the HC surface were characterized by slightly larger FAs $\left(\bar{\ell}_{H C}=4.63 \mu \mathrm{m}, \mathrm{P}<0.05\right)$ than those on the NT1 surface. Although the inter-tube spacing of the former $(\sim 25 \mathrm{~nm})$ is below the optimal range for the establishment of FAs, this interesting finding may be explained by postulating a beneficial contribution of the additional 

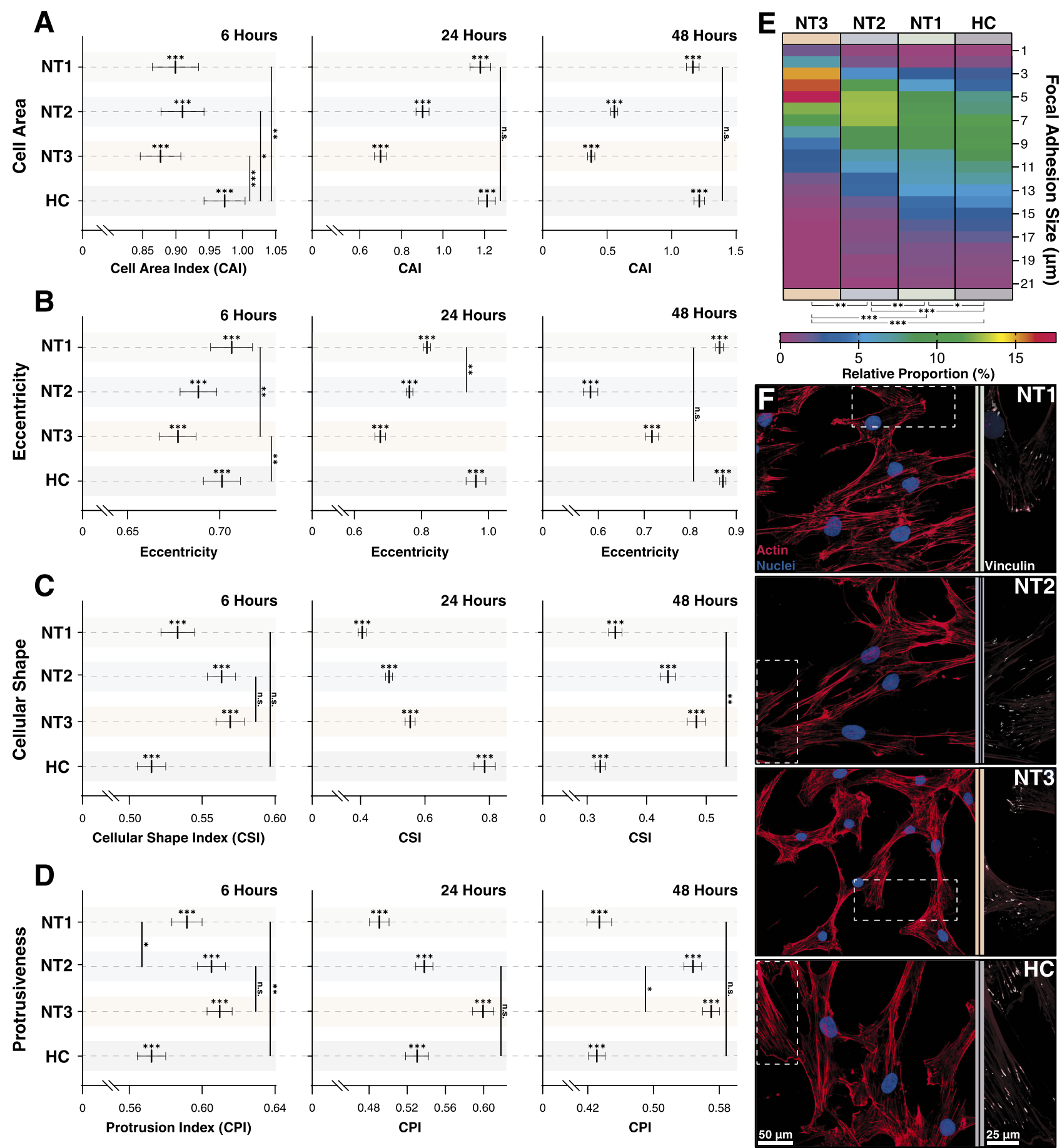

Figure 6 Morphological characterization of hMSCs including (A) cell area index, (B) eccentricity, (C) cell shape index and (D) protrusion index at 6 hrs, 24 hrs and 48 hrs. (E) Distribution of focal adhesion length with respect to relative proportion. Cells on the NT3 surface have the lowest proportion of FAs above II $\mu \mathrm{m}$ in length. (F) representative fluorescence images. Statistical significance was defined as $*=P<0.05, * *=P<0.01, * * *=P<0.001$ and n.s. $=$ non-significant.

surface provided by the enveloping L-HC layer $(\sim 33 \mathrm{~nm})$, which would thus add extra area for the formation of FAs.

\section{Osterix (OSX)}

In addition to the effects on cell adhesion and proliferation, surface nanotopography is known for its ability to affect the differentiation of multipotent stem cells towards a specific fate. ${ }^{14,86,87}$ Topographical modification of titanium has shown to be effective in lineage guidance, ${ }^{88-91}$ with $\mathrm{TiO}_{2}$ nanotubes being capable of promoting variant strengths of osteogenic differentiation as a function of key physical properties of the tubular structures (e.g., diameter, length). ${ }^{24,25,30,32} \mathrm{In}$ 

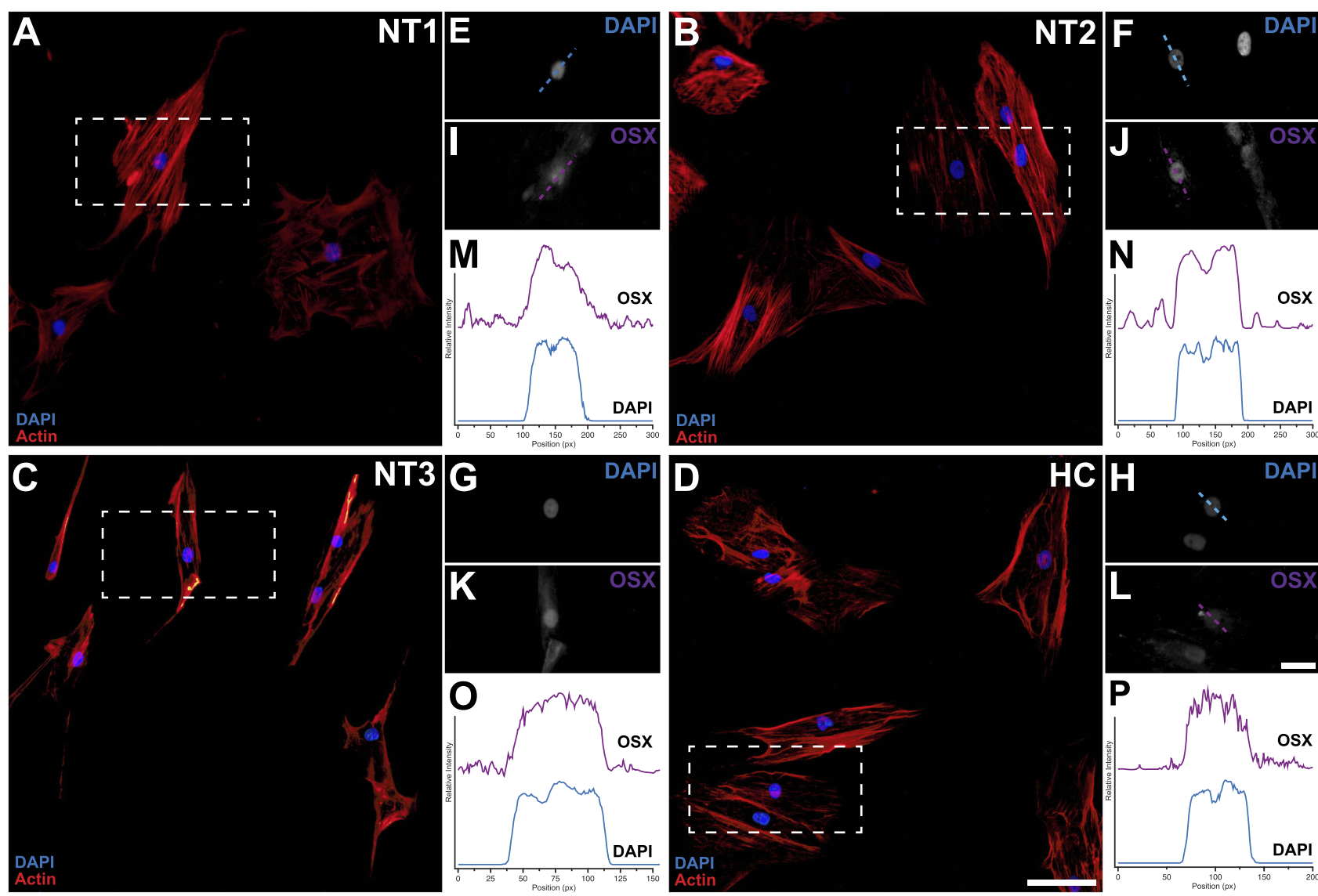

Figure 7 Signalling of osteogenic differentiation through nuclear localization of Osterix (OSX/Sp7) at 7 days. Each set of 4 panels presents cell body, 2D-position and intensity profile of the nucleus (DAPI) and OSX signal for hMSCs on (A-D) NTI, (E-H) NT2, (I-L) NT3 and (M-P) HC surfaces.

this context, we investigated the osteoinductive capabilities of nanotubular architectures through the identification and distribution of the zinc-finger-containing Osterix/Sp7 (OSX) protein. $^{92}$ Regulation of the OSX gene by the osteoblastic RUNX2 transcription factor, ${ }^{93}$ coupled with its control of downstream osteoblastic genes (e.g., Osteocalcin, Type I Collagen), ${ }^{92}$ affirms its importance for the commitment of preosteoblastic cells toward mature osteoblasts. Specifically, the nuclear localization of OSX is an indication of the commitment to the osteogenic lineage. ${ }^{92,94}$ Using a fluorescent reporter for OSX $\left(\lambda_{\mathrm{em}}=665 \mathrm{~nm}\right)$, and without the use of differentiation media, we were able to detect the presence and localization of the OSX signal to the nuclear region as identified by a DAPI counterstain $\left(\lambda_{\mathrm{em}}=461 \mathrm{~nm}\right)$, shown in Figure 7. The confirmation of the osteoblastic commitment reaffirms that surface-driven osteogenic induction is taking place on all surfaces and thereby sets the groundwork for subsequent analyses (i.e., Raman) that asses the quality of the bone that is deposited by cells on their respective conditions.

\section{Mineral Analysis}

To complete our analysis of hMSC response to nanotubular surfaces, we analyzed the properties of mineral nodules deposited in situ at 28 days of culture without the use of differentiation media by analyzing their Raman signature (Figure 8A) using four parameters were assessed as a way to gain insight into specific substrate-induced variations of bone quality. ${ }^{55}$ The first parameter we considered was the mineral-to-matrix ratio (Figure $8 \mathrm{~B}$ ), which normalizes the amount of bone against the quantity of collagen, and has been shown to increase with bone tissue age. ${ }^{54,55}$ In our case, the mineral nodules deposited on the $\mathrm{HC}$ architecture showed the highest ratio with a marked difference with the NT1, while NT2 and NT3 did not show any statistical distinction between them. We successively assessed the carbonate-to-phosphate ratio (Figure 8C), which is indicative of regularly occurring carbonate substitutions in hydroxyapatite, a phenomenon was shown to increase with tissue age and to depend on the bone architecture and mineral 

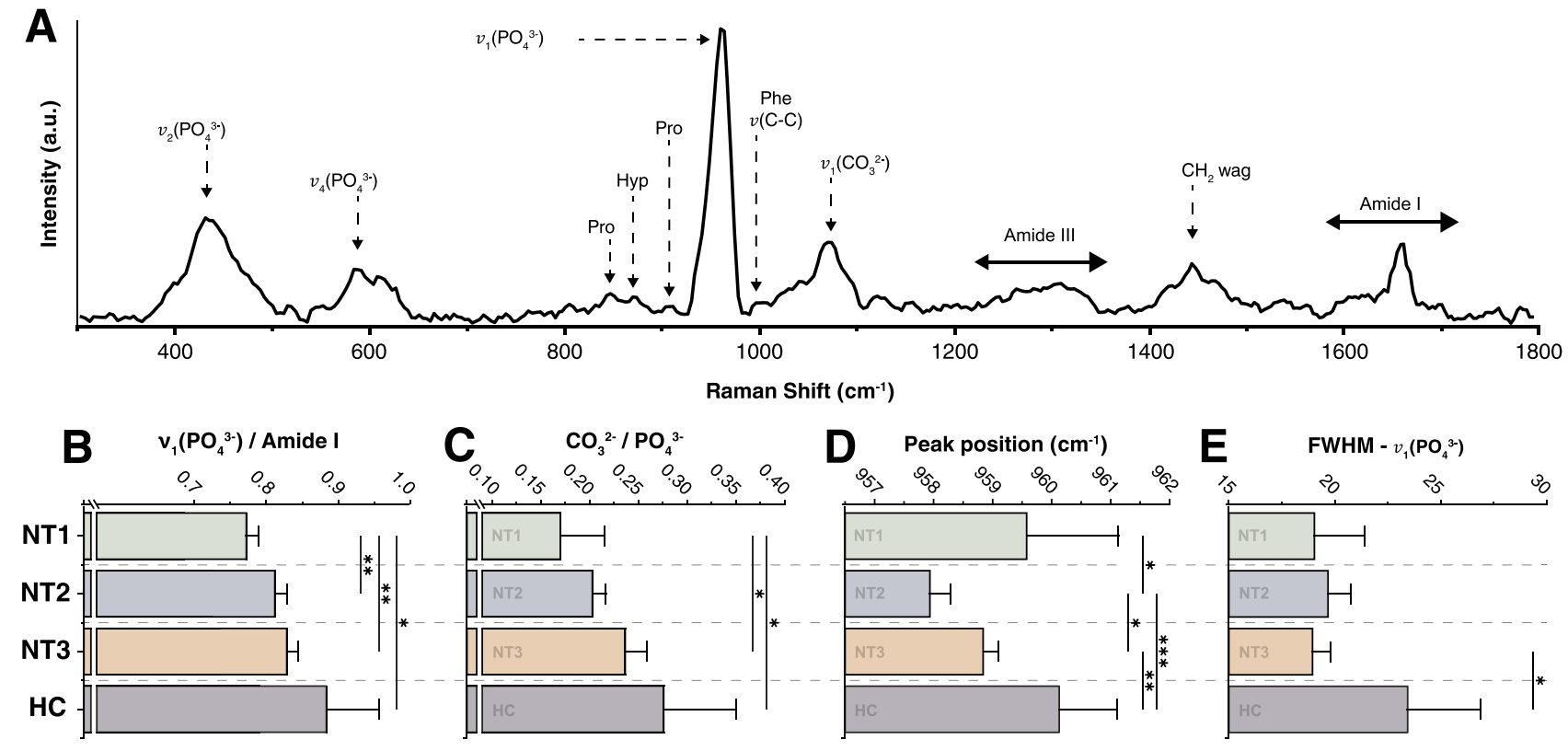

Figure 8 (A) Representative Raman spectrum acquired on deposited bone mineral after 28 days of culture. Quantification of physicochemical parameters on nodules deposited on NTI $(n=5), N T 2(n=10), N T 3(n=6)$ and HC $(n=12)$ surfaces. Mean values with bars representing $95 \%$ Cl for the (B) Mineral-to-matrix ratio, $(\mathbf{C})$ Carbonate-to -phosphate ratio, (D) Peak position and (E) I/FWHM of the phosphate $\left(\mathrm{PO}_{4}{ }^{3-}\right)$ vI band. Statistical significance was defined as $*=\mathrm{P}<0.05, * *=\mathrm{P}<0.0 \mathrm{I}$ and $* * *=\mathrm{P}<0.00 \mathrm{I}$.

crystallinity. ${ }^{54,55}$ In these cases, the variability for the $\mathrm{HC}$ surface is greater than that for the other conditions, and this may be either due to chemical inhomogeneities and/or to the fact that the carbonate band partially overlaps another phosphate band at $\sim 1076 \mathrm{~cm}^{-1}$, thereby reducing measurement precision especially for apatites with minimal carbonate content. $^{54,55}$ Considering that significant differences were nonetheless detected between nodules deposited on the HC substrates and those on the NT1 surface, and between nodules on NT3 surface and those on the NT1 substrate, by taking into context the mineral-to-matrix and phosphateto-mineral ratios, we can conclude that among the conditions investigated, more advanced mineralization was found on the HC and NT3 substrates. The third parameter analyzed was the position of the $v_{1} \mathrm{PO}_{4}{ }^{3-}$ band within the 955-962 $\mathrm{cm}^{-1}$ range. Previous work has demonstrated that the transition of the peak position from lower to higher wavenumbers, and a band shape change from a broad to narrow, are associated with the transformation of disordered amorphous calcium phosphate (ACP, $950 \mathrm{~cm}^{-1}$ ) to mature crystalline hydroxyapatite (HAP, 960-962 cm ${ }^{-1}$ ). ${ }^{62,95}$ Raman data from all surfaces exhibit peaks in the neighbourhood of $958 \mathrm{~cm}^{-1}$ (Figure 8D), indicating that the minerals are mainly composed of transformed HAP, with potential inclusions of its transitional intermediates (e.g., octacalciumphosphate $[\mathrm{OCP}])^{61}$ in those conditions that exhibited the highest shift. In particular, nodules on NT1 and $\mathrm{HC}$ surfaces presented with a statistically similar peak position indicating a greater shared predominance of crystalline HAP when compared to the red-shifted bands exhibited on NT2 and NT3. Lastly, we assessed the inverse of full-width-at-half-maximum (1/FWHM) of the $v_{1} \mathrm{PO}_{4}{ }^{3-}$ band (Figure $8 \mathrm{E}$ ) as a measure of crystallinity and crystalline homogeneity. The transition from disordered ACP to ordered HAP is observed with increased crystallite $c$-axis length and improvement in the stoichiometric organization of the crystal lattice. ${ }^{54,96}$ Subsequently, reduction in shortrange disorder and progressive homogeneity of the mineral constituents have shown to result in a narrowing of the bandwidth, ${ }^{60}$ as observed for the $\mathrm{HC}$ architecture with respect to the NT conditions. Taken together, our Raman results show that the $\mathrm{HC}$ architecture induces more advanced mineralization of crystalline hydroxyapatite nodules when compared to the other conditions. Notably, the mineralization in the crystalline hydroxyapatite nodules on NT1 lags behind. Conversely, the hydroxyapatite on the NT3 surface is characterized by a degree of mineralization comparable to that of the $\mathrm{HC}$ substrate, but with a significantly lower degree of crystallinity and/or crystalline homogeneity. In this context, the correlation between early morphological signatures, namely MSC cell number and cell area at short intervals, with osteogenesis 
assessment was previously demonstrated, highlighting the power of immunofluorescence imaging to predict long-term mineralization. ${ }^{97}$ It was shown that cell morphology, in particular, cell area, after 3 days was highly correlated with 35-day mineralization. ${ }^{97}$ We can thus infer that the greater cell areas determined on the NT1 and HC surfaces at $48 \mathrm{~h}$ may be responsible for the faster formation of crystalline HPA, with a more advanced maturation stage (in terms of crystalline homogeneity) for the latter. On the other hand, previous work has also reported that osteoblasts cultured on $100 \mathrm{~nm}$ diameter nanotubes experience increased nuclear elongation, a factor which was associated with increased alkaline phosphatase (ALP) and osteocalcin expression, as well as greater bone-forming ability in vivo, ${ }^{49}$ and that, in part, could further explain the results obtained for the NT3 surface.

\section{Conclusion}

In this work, we created differentially sized nanotubular patterns (NTs) through the rational modulation of the experimental parameters, which also permitted us to create a two-tiered nanotubular architecture (HC). Through the characterization of the complex nanotopographical environment of these surfaces, we delineated important new connections between nanotubes and stem cell response (including proliferation, morphology and deposition of bone mineral). Ultimately, results from this work strengthen the role of direct physicochemical cueing to unlock the potential to affect healing and controlling stem cell fate, paving the way to combine both physical and chemical cues in a synergistic manner for drastically improved, efficient and effective biological outcomes of implantable materials. In this context, despite the advances made possible by the development of new technologies, the progress of nanoarchitectures for biomedical applications is still restricted by the technical capability of design and fabrication methods, often inadequate to rapidly replicate micro- and nano-scale patterns on relatively large surface areas of medically relevant materials for high-throughput manufacturing. ${ }^{98}$ While anodization confers the flexibility of controlling the diameter, we have demonstrated that other morphological parameters are equally important, in particular, the degree of order. The creation of the HC nanoarchitecture could thus become a strategy to vary the order of nanotube arrays without changing their diameter, thereby becoming allowing one extra level of synergistic control of the resulting nanotopography.

\section{Acknowledgments}

This work was supported by the Natural Sciences and Engineering Research Council of Canada (NSERC) through the Discovery grant, by the Canada Foundation for Innovation (CFI) and the Ontario Ministry of Research and Innovation (MRI) through the Leaders of Opportunity (LOF) fund. FV also acknowledges the IBEC's Severo Ochoa Visiting program for external outstanding researchers. The authors acknowledge the resources and assistance provided by the Cell Biology and Image Acquisition (CBIA) Core, funded in partnership by the University of Ottawa and CFI.

\section{Disclosure}

These authors report no conflicts of interest in this work.

\section{References}

1. Jeon HJ, Simon CG, Kim GH. A mini-review: cell response to microscale, nanoscale, and hierarchical patterning of surface structure. J Biomed Mater Res - Part B Appl Biomater. 2014;102:1580-1594. doi:10.1002/jbm.b.v102.7

2. Zhang Y, Gordon A, Qian W, Chen W. Engineering nanoscale stem cell niche: direct stem cell behavior at cell-matrix interface. $A d v$ Healthc Mater. 2015;4:1900-1914. doi:10.1002/adhm.201500351

3. Zhukova Y, Skorb EV. Cell guidance on nanostructured metal based surfaces. Adv Healthc Mater. 2017;6:1600914. doi:10.1002/adhm. 201600914

4. Hogrebe NJ, Reinhardt JW, Gooch KJ. Biomaterial microarchitecture: a potent regulator of individual cell behavior and multicellular organization. J Biomed Mater Res Part A. 2017;105:640-661. doi:10. 1002/jbm.a.35914

5. Wang S, Li J, Zhou Z, Zhou S, Hu Z. Sphingosine-1-phosphate improves the biological features of mouse bone marrow-derived EPCs partially through PI3K/AKT/eNOS/NO pathway. Molecules. 2019;24:1-13.

6. Limongi T, Tirinato L, Pagliari F, et al. Fabrication and applications of micro/nanostructured devices for tissue engineering. Nano-Micro Letters. 2017;9:1-13. doi:10.1007/s40820-016-0103-7

7. Das RK, Zouani OF. A review of the effects of the cell environment physicochemical nanoarchitecture on stem cell commitment. Biomaterials. 2014;35:5278-5293. doi:10.1016/j.biomaterials.2014.03.044

8. Boyan BD, Lotz EM, Schwartz Z. Roughness and hydrophilicity as osteogenic biomimetic surface properties. Tissue Eng Part A. 2017;23:1479-1489. doi:10.1089/ten.tea.2017.0048

9. Simitzi C, Ranella A, Stratakis E. Controlling the morphology and outgrowth of nerve and neuroglial cells: the effect of surface topography. Acta Biomaterialia. 2017;51:21-52. doi:10.1016/j. actbio.2017.01.023

10. Chen Z, Bachhuka A, Wei F, et al. Nanotopography-based strategy for the precise manipulation of osteoimmunomodulation in bone regeneration. Nanoscale. 2017;9:18129-18152. doi:10.1039/ C7NR05913B

11. Chen S, Guo Y, Liu R, et al. Tuning surface properties of bone biomaterials to manipulate osteoblastic cell adhesion and the signaling pathways for the enhancement of early osseointegration. Colloids Surfaces B Biointerfaces. 2018;164:58-69. doi:10.1016/j.colsurfb.2018.01.022 
12. Ermis M, Antmen E, Hasirci V. Micro and Nanofabrication methods to control cell-substrate interactions and cell behavior: a review from the tissue engineering perspective. Bioact Mater. 2018;3:355-369. doi:10.1016/j.bioactmat.2018.05.005

13. Greiner AM, Sales A, Chen H, Biela SA, Kaufmann D, Kemkemer R. Nano- and microstructured materials for in vitro studies of the physiology of vascular cells. Beilstein J Nanotechnol. 2016;7:1620-1641. doi:10.3762/bjnano.7.155

14. Krishna L, Dhamodaran K, Jayadev C, et al. Nanostructured scaffold as a determinant of stem cell fate. Stem Cell Res Ther. 2016;7:1-12. doi:10.1186/s13287-016-0440-y

15. Dobbenga S, Fratila-Apachitei LE, Zadpoor AA. Nanopatterninduced osteogenic differentiation of stem cells - a systematic review. Acta Biomaterialia. 2016;46:3-14. doi:10.1016/j.actbio.2016. 09.031

16. Di Cio S, Gautrot JE. Cell sensing of physical properties at the nanoscale: mechanisms and control of cell adhesion and phenotype. Acta Biomaterialia. 2016;30:26-48. doi:10.1016/j.actbio.2015.11.027

17. Park J, Bauer S, Pittrof A, Killian MS, Schmuki P, Von der Mark K. Synergistic control of mesenchymal stem cell differentiation by nanoscale surface geometry and immobilized growth factors on TiO2 nanotubes. Small. 2012;8:98-107. doi:10.1002/smll.201100790

18. Tan AW, Pingguan-Murphy B, Ahmad R, Akbar SA. Review of titania nanotubes: fabrication and cellular response. Ceram Int. 2012;38:4421-4435. doi:10.1016/j.ceramint.2012.03.002

19. Fu Y, Mo A. A review on the electrochemically self-organized titania nanotube arrays: synthesis, modifications, and biomedical applications. Nanoscale Res Lett. 2018;13(1):187. doi:10.1186/s11671-018-2597-z

20. Minagar S, Wang J, Berndt CC, Ivanova EP, Wen C. Cell response of anodized nanotubes on titanium and titanium alloys. J Biomed Mater Res Part A. 2013;101(A):2726-2739. doi:10.1002/jbm.a.v101a.9

21. Brammer KS, Frandsen CJ, Jin S. TiO2 nanotubes for bone regeneration. Trends Biotechnol. 2012;30:315-322. doi:10.1016/j. tibtech.2012.02.005

22. Khudhair D, Bhatti A, Li Y, et al. Anodization parameters influencing the morphology and electrical properties of $\mathrm{TiO} 2$ nanotubes for living cell interfacing and investigations. Mater Sci Eng C. 2016;59:1125-1142. doi:10.1016/j.msec.2015.10.042

23. Bai L, Yang Y, Mendhi J, et al. The effects of TiO 2 nanotube arrays with different diameters on macrophage/endothelial cell response and ex vivo hemocompatibility. J Mater Chem B. 2018;6:6322-6333. doi:10.1039/C8TB01675E

24. Park J, Bauer S, Schlegel KA, Neukam FW, von der Mark K, Schmuki P. TiO 2 nanotube surfaces: $15 \mathrm{~nm}$-an optimal length scale of surface topography for cell adhesion and differentiation. Small. 2009;5:666-671. doi:10.1002/smll.v5:6

25. Park J, Bauer S, Von Der Mark K, Schmuki P. Nanosize and vitality: TIO2 nanotube diameter directs cell fate. Nano Lett. 2007;7:1686-1691. doi:10.1021/nl070678d

26. Cowden K, Dias-Netipanyj MF, Popat KC. Nanomedicine Nanotechnology. Biol Med. 2019;17:380-390.

27. von der Mark K, Bauer S, Park J, Schmuki P. Another look at "Stem cell fate dictated solely by altered nanotube dimension". Proc Natl Acad Sci. 2009;106:E60-E60. doi:10.1073/pnas.0903663106

28. Oh S, Brammer KS, Li YSJ, et al. Stem cell fate dictated solely by altered nanotube dimension. Proc Natl Acad Sci USA 2009;106:2130-2135.

29. Li M, Yang Y. Nanoscale $\mathrm{TiO}_{2}$ nanotubes as a basis for governing cell behaviors and application challenges. Int $J$ Nanomedicine. 2017;12:575-576. doi:10.2147/IJN

30. Oh S, Brammer KS, Li YSJ, et al. Stem cell fate dictated solely by altered nanotube dimension. Proc Natl Acad Sci USA. 2009;106:2130-2135. doi:10.1073/pnas.0813200106

31. Wang J, Meng F, Song W, et al. Nanostructured titanium regulates osseointegration via influencing macrophage polarization in the osteogenic environment. Int J Nanomedicine. 2018;13:4029-4043. doi:10.2147/IJN
32. Lv L, Liu Y, Zhang P, et al. The nanoscale geometry of TiO2 nanotubes influences the osteogenic differentiation of human adipose-derived stem cells by modulating H3K4 trimethylation. Biomaterials. 2015;39: 193-205. doi:10.1016/j.biomaterials.2014.11.002

33. Yu W, Qian C, Jiang X, Zhang F, Weng W. Mechanisms of stem cell osteogenic differentiation on TiO 2 nanotubes. Colloids Surfaces $B$ Biointerfaces. 2015;136:779-785. doi:10.1016/j.colsurfb.2015. 10.019

34. Biggs MJP, Richards RG, Gadegaard N, Wilkinson CDW, Dalby MJ. The effects of nanoscale pits on primary human osteoblast adhesion formation and cellular spreading. $J$ Mater Sci Mater Med. 2007;18:399-404. doi:10.1007/s10856-006-0705-6

35. Dalby MJ, Gadegaard N, Tare R, et al. The control of human mesenchymal cell differentiation using nanoscale symmetry and disorder. Nat Mater. 2007;6:997-1003. doi:10.1038/nmat2013

36. Li S, Zhang G, Guo D, Yu L, Zhang W. Anodization fabrication of highly ordered TiO2 nanotubes. J Phys Chem C. 2009;113:12759-12765. doi:10.1021/jp903037f

37. Yu D, Song Y, Zhu X, Yang R, Han A. Morphological evolution of $\mathrm{TiO} 2$ nanotube arrays with lotus-root-shaped nanostructure. Appl Surf Sci. 2013;276:711-716. doi:10.1016/j.apsusc.2013.03.158

38. Jin R, Fan H, Liu Y, et al. Formation mechanism of lotus-root-shaped nanostructure during two-step anodization. Electrochim Acta. 2016;188:421-427. doi:10.1016/j.electacta.2015.12.027

39. Sitler SJ, Raja KS. Self-ordering dual-layered honeycomb nanotubular titania: a study in formation mechanisms. RSC Adv. 2016;6:11991-12002. doi:10.1039/C5RA24667A

40. Paterlini TT, Nogueira LFB, Tovani CB, Cruz MAE, Derradi R, Ramos AP. The role played by modified bioinspired surfaces in interfacial properties of biomaterials. Biophys Rev. 2017;9:683-698. doi:10.1007/s12551-017-0306-2

41. Liu X, Liu R, Cao B, et al. Subcellular cell geometry on micropillars regulates stem cell differentiation. Biomaterials. 2016;111:27-39. doi:10.1016/j.biomaterials.2016.09.023

42. Fan R, Wan J. Electrode distance regulates the anodic growth of titanium dioxide (TiO2) nanotubes. Nanotechnology. 2017;28 (25):25LT01. doi:10.1088/1361-6528/aa703d

43. Wang D, Yu B, Wang C, Zhou F, Liu W. A novel protocol toward perfect alignment of anodized $\mathrm{TiO} 2$ nanotubes. Adv Mater. 2009;21:1964-1967. doi:10.1002/adma.v21:19

44. Schneider CA, Rasband WS, Eliceiri KW. NIH image to imageJ: 25 years of image analysis. Nat Methods. 2012;9:671-675. doi:10.1038/ nmeth.2089

45. Carpenter AE, Jones TR, Lamprecht MR, et al. CellProfiler: image analysis software for identifying and quantifying cell phenotypes. Genome Biol. 2006;7:R100. doi:10.1186/gb-2006-7-10-r100

46. Horcas I, Fernández R, Gómez-Rodríguez JM, Colchero J, GómezHerrero J, Baro AM. WSXM: a software for scanning probe microscopy and a tool for nanotechnology. Rev Sci Instrum. 2007;78:013705. doi:10.1063/1.2432410

47. Bormashenko E, Frenkel M, Vilk A, et al. WSXM: a software for scanning probe microscopy and a tool for nanotechnology. Entropy. 2018;20:1-18

48. Steyer A, Guenoun P, Beysens D, Knobler CM. Two-dimensional ordering during droplet growth on a liquid surface. Phys Rev B. 1990;42:1086-1089. doi:10.1103/PhysRevB.42.1086

49. Brammer KS, Oh S, Cobb CJ, Bjursten LM, van der Heyde H, Jin S. Improved bone-forming functionality on diameter-controlled $\mathrm{TiO} 2$ nanotube surface. Acta Biomaterialia. 2009;5:3215-3223. doi:10.1016/j. actbio.2009.05.008

50. Farlay D, Panczer G, Rey C, Delmas PD, Boivin G. Mineral maturity and crystallinity index are distinct characteristics of bone mineral. J Bone Miner Metab. 2010;28:433-445. doi:10.1007/s00774-009-0146-7

51. Versaevel M, Grevesse T, Gabriele S. Spatial coordination between cell and nuclear shape within micropatterned endothelial cells. Nat Commun. 2012;3:671. doi:10.1038/ncomms1668 
52. Hardcastle FD, Ishihara H, Sharma R, Biris AS. Photoelectroactivity and Raman spectroscopy of anodized titania (TiO2) photoactive water-splitting catalysts as a function of oxygen-annealing temperature. J. Mater. Chem. 2011;21:6337-6345. doi:10.1039/c0jm03106b

53. Penel G, Leroy G, Rey C, Bres E. MicroRaman spectral study of the PO 4 and CO 3 vibrational modes in synthetic and biological apatites. Calcif Tissue Int. 1998;63:475-481. doi:10.1007/ s002239900561

54. Mandair GS, Morris MD. Contributions of Raman spectroscopy to the understanding of bone strength. Bonekey Rep. 2015;4:1-8. doi:10.1038/bonekey.2014.115

55. Morris MD, Mandair GS. Raman assessment of bone quality. Clin Orthop Relat Res. 2011;469:2160-2169. doi:10.1007/s11999-010-1692-y

56. Awonusi A, Morris MD, Tecklenburg MMJ. Carbonate assignment and calibration in the raman spectrum of apatite. Calcif Tissue Int. 2007;81:46-52. doi:10.1007/s00223-007-9034-0

57. Carden A, Morris MD. Application of vibrational spectroscopy to the study of mineralized tissues (review). J Biomed Opt. 2000;5:259. doi:10.1117/1.429994

58. Morris MD, Finney WF. Recent developments in Raman and infrared spectroscopy and imaging of bone tissue. Spectroscopy. 2004;18: 155-159. doi:10.1155/2004/765753

59. Wopenka B, Pasteris JD. A mineralogical perspective on the apatite in bone. Mater Sci Eng C. 2005;25:131-143. doi:10.1016/j. msec.2005.01.008

60. Alakpa EV, Burgess KEV, Chung P, et al. Nacre topography produces higher crystallinity in bone than chemically induced osteogenesis. ACS Nano. 2017;11:6717-6727. doi:10.1021/acsnano. $7 \mathrm{~b} 01044$

61. Crane NJ, Popescu V, Morris MD, Steenhuis P, Ignelzi MA. Raman spectroscopic evidence for octacalcium phosphate and other transient mineral species deposited during intramembranous mineralization. Bone. 2006;39:434-442. doi:10.1016/j.bone.2006.02.059

62. Stewart S, Shea DA, Tarnowski CP, et al. Trends in early mineralization of murine calvarial osteoblastic cultures: a Raman microscopic study. J Raman Spectrosc. 2002;33:536-543. doi:10.1002/(ISSN) $1097-4555$

63. Massey FJ. The Kolmogorov-Smirnov test for goodness of fit. $J$ Am Stat Assoc. 1951;46:68-78. doi:10.1080/01621459.1951.10500769

64. Ruxton GD. The unequal variance t-test is an underused alternative to Student's t-test and the Mann-Whitney U test. Behav Ecol. 2006;17:688-690. doi:10.1093/beheco/ark016

65. Biggs MJP, Richards RG, Dalby MJ. Nanomedicine nanotechnology. Biol Med. 2010;6:619-633.

66. Huang J, Gräter SV, Corbellini F, et al. Impact of order and disorder in RGD nanopatterns on cell adhesion. Nano Lett. 2009;9:1111-1116. doi:10.1021/nl $1803548 \mathrm{~b}$

67. Ngandu Mpoyi E, Cantini M, Reynolds PM, Gadegaard N, Dalby MJ, Salmerón-Sánchez M. Protein adsorption as a key mediator in the nanotopographical control of cell behavior. ACS Nano. 2016;10:6638-6647. doi:10.1021/acsnano.6b01649

68. Yu WQ, Jiang XQ, Zhang FQ, Xu L. The effect of anatase TiO2 nanotube layers on MC3T3-E1 preosteoblast adhesion, proliferation, and differentiation. J Biomed Mater Res Part A. 2010;94:1012-1022.

69. Tsuchiya H, Macak JM, Müller L, et al. Hydroxyapatite growth on anodic TiO2 nanotubes. $J$ Biomed Mater Res Part A. 2006;77:534-541. doi:10.1002/(ISSN)1552-4965

70. Su Z, Zhang L, Jiang F, Hong M. Formation of crystalline TiO2 by anodic oxidation of titanium. Prog Nat Sci Mater Int. 2013;23:294-301. doi:10.1016/j.pnsc.2013.04.004

71. Gui N, Xu W, Myers DE, Shukla R, Tang HP, Qian M. The effect of ordered and partially ordered surface topography on bone cell responses: a review. Biomater Sci. 2018;6:250-264. doi:10.1039/C7BM01016H

72. Yi JH, Bernard C, Variola F, et al. Characterization of a bioactive nanotextured surface created by controlled chemical oxidation of titanium. Surf Sci. 2006;600:4613-4621. doi:10.1016/j.susc.2006.07.053
73. Wang X, Liu H, Zhu M, et al. Mechanical stability of the cell nucleusroles played by the cytoskeleton in nuclear deformation and strain recovery. J Cell Sci. 2017;131:jcs209627.doi:10.1242/jcs.209627

74. Tomakidi P, Schulz S, Proksch S, Weber W, Steinberg T. Focal adhesion kinase (FAK) perspectives in mechanobiology: implications for cell behaviour. Cell Tissue Res. 2014;357:515-526. doi:10.1007/ s00441-014-1945-2

75. Biggs MJP, Dalby MJ. Focal adhesions in osteoneogenesis. Proc Institut Mech Eng Part H J Eng Med. 2010;224:1441-1453. doi:10.1243/ 09544119JEIM775

76. Nagano M, Hoshino D, Koshikawa N, Akizawa T, Seiki M. Turnover of focal adhesions and cancer cell migration. Int J Cell Biol. 2012.

77. Tsimbouri P, Gadegaard N, Burgess K, et al. Nanotopographical effects on mesenchymal stem cell morphology and phenotype. J Cell Biochem. 2014;115:380-390. doi:10.1002/jcb.24673

78. Schlie S, Gruene M, Dittmar H, Chichkov BN. Dynamics of cell attachment: adhesion time and force. Tissue Eng Part C Methods. 2012;18:688-696. doi:10.1089/ten.tec.2011.0635

79. Kulangara K, Yang Y, Yang J, Leong KW. Nanotopography as modulator of human mesenchymal stem cell function. Biomaterials. 2012;33:4998-5003. doi:10.1016/j.biomaterials.2012.03.053

80. Kim DH, Wirtz D. Focal adhesion size uniquely predicts cell migration. FASEB J. 2013;27:1351-1361. doi:10.1096/fj.12-220160

81. Cao X, Ban E, Baker BM, et al. Multiscale model predicts increasing focal adhesion size with decreasing stiffness in fibrous matrices. Proc Natl Acad Sci U S A. 2017;114:E4549-E4555. doi:10.1073/pnas.1620486114

82. Legerstee K, Geverts B, Slotman JA, Houtsmuller AB. http://Dynamics and distribution of paxillin, vinculin, zyxin and VASP depend on focal adhesion location and orientation. Sci Rep. 2019;9(1):1-8. doi:10.1038/ s41598-019-46905-2

83. Liu J, Wang Y, Goh WI, et al. Talin determines the nanoscale architecture of focal adhesions. Proc Natl Acad Sci U S A. 2015;112:E4864-E4873. doi:10.1073/pnas. 1512025112

84. Humphries JD, Wang P, Streuli C, Geiger B, Humphries MJ, Ballestrem C. Vinculin controls focal adhesion formation by direct interactions with talin and actin. J Cell Biol. 2007;179:1043-1057. doi:10.1083/jcb.200703036

85. Bauer S, Park J, von der Mark K, Schmuki P. Improved attachment of mesenchymal stem cells on super-hydrophobic $\mathrm{TiO} 2$ nanotubes. Acta Biomaterialia. 2008;4:1576-1582. doi:10.1016/j. actbio.2008.04.004

86. Teo BKK, Wong ST, Lim CK, et al. Nanotopography modulates mechanotransduction of stem cells and induces differentiation through focal adhesion kinase. ACS Nano. 2013;7:4785-4798. doi:10.1021/nn304966z

87. Niu H, Lin D, Tang W, et al. Surface topography regulates osteogenic differentiation of MSCs via crosstalk between FAK/MAPK and ILK/ $\beta$ catenin pathways in a hierarchically porous environment. ACS Biomater Sci Eng. 2017;3:3161-3175. doi:10.1021/acsbiomaterials.7b00315

88. Cowden K, Dias-Netipanyj MF, Popat KC. Adhesion and proliferation of human adipose-derived stem cells on titania nanotube surfaces. Regen Eng Transl Med. 2019;5(4):435-45.doi:10.1007/ s40883-019-00091-9

89. Lai M, Cai K, Hu Y, Yang X, Liu Q. Regulation of the behaviors of mesenchymal stem cells by surface nanostructured titanium. Colloids Surfaces B. 2012;97:211-220. doi:10.1016/j. colsurfb.2012.04.029

90. Lavenus S, Berreur M, Trichet V, Pilet P, Louarn G, Layrolle P. Adhesion and osteogenic differentiation of human mesenchymal stem cells on titanium nanopores. Eur Cells Mater. 2011;22:84-96. doi:10.22203/eCM

91. Shin YC, Pang KM, Han DW, et al. Enhanced osteogenic differentiation of human mesenchymal stem cells on Ti surfaces with electrochemical nanopattern formation. Mater Sci Eng C. 2019;99:1174-1181. doi:10.1016/j.msec.2019.02.039 
92. Nakashima K, Zhou $X$, Kunkel $G$, et al. The novel zinc finger-containing transcription factor osterix is required for osteoblast differentiation and bone formation. Cell. 2002;108:17-29. doi:10.1016/S0092-8674(01)00622-5

93. Nishio Y, Dong Y, Paris M, O’Keefe RJ, Schwarz EM, Drissi H. Runx2-mediated regulation of the zinc finger Osterix/Sp7 gene. Gene. 2006;372:62-70. doi:10.1016/j.gene.2005.12.022

94. Artigas N, Ureña C, Rodríguez-Carballo E, Rosa JL, Ventura F. Mitogenactivated protein kinase (MAPK)-regulated interactions between osterix and runx2 are critical for the transcriptional osteogenic program. $J$ Biol Chem. 2014;289:27105-27117. doi:10.1074/jbc.M114.576793

95. Stammeier JA, Purgstaller B, Hippler D, Mavromatis V, Dietzel M. Methods. 2018;5:1241-1250.
96. Yerramshetty JS, Lind C, Akkus O. The compositional and physicochemical homogeneity of male femoral cortex increases after the sixth decade. Bone. 2006;39:1236-1243. doi:10.1016/j.bone.2006.06.002

97. Marklein RA, Lo Surdo JL, Bellayr IH, Godil SA, Puri RK, Bauer SR. High content imaging of early morphological signatures predicts long term mineralization capacity of human mesenchymal stem cells upon osteogenic induction. Stem Cells. 2016;34:935-947. doi:10.1002/stem.v34.4

98. Magin CM, Alge DL, Anseth KS. Bio-inspired 3D microenvironments: a new dimension in tissue engineering. Biomedical Materials. Biomed Mater. 2016;11(2):022001. doi:10.1088/1748-6041/11/2/022001
International Journal of Nanomedicine

\section{Publish your work in this journal}

The International Journal of Nanomedicine is an international, peerreviewed journal focusing on the application of nanotechnology in diagnostics, therapeutics, and drug delivery systems throughout the biomedical field. This journal is indexed on PubMed Central, MedLine, CAS, SciSearch ${ }^{\mathbb{B}}$, Current Contents ${ }^{\mathbb{B}} /$ Clinical Medicine, $^{2}$
Dovepress

Journal Citation Reports/Science Edition, EMBase, Scopus and the Elsevier Bibliographic databases. The manuscript management system is completely online and includes a very quick and fair peer-review system, which is all easy to use. Visit http://www.dovepress.com/ testimonials.php to read real quotes from published authors. 